\title{
MULTIPLE SOLUTIONS FOR NONLINEAR DIRICHLET PROBLEMS WITH CONCAVE TERMS
}

\author{
LESZEK GASIŃSKI and NIKOLAOS S. PAPAGEORGIOU*
}

\begin{abstract}
We consider a nonlinear parametric Dirichlet problem with parameter $\lambda>0$, driven by the $p$ Laplacian and with a concave term $\lambda|u|^{q-2} u, 1<q<p$ and a Carathéodory perturbation $f(z, \zeta)$ which is asymptotically $(p-1)$-linear at infinity. Using variational methods combined with Morse theory and truncation techniques, we show that there is a critical value $\lambda^{*}>0$ of the parameter such that for $\lambda \in\left(0, \lambda^{*}\right)$ the problem has five nontrivial smooth solutions, four of constant sign (two positive and two negative) and the fifth nodal. In the semilinear case $(p=2)$, we show that there is a sixth nontrivial smooth solution, but we cannot provide information about its sign. Finally for the critical case $\lambda=\lambda^{*}$, we show that the nonlinear problem $(p \neq 2)$ still has two nontrivial constant sign smooth solutions and the semilinear problem $(p=2)$ has three nontrivial smooth solutions, two of which have constant sign.
\end{abstract}

\section{Introduction}

Let $\Omega \subseteq \mathrm{R}^{N}$ be a bounded domain with $C^{2}$-boundary $\partial \Omega$. In this paper we study the following nonlinear parametric Dirichlet problem:

$$
\left\{\begin{array}{l}
-\Delta_{p} u(z)=\lambda|u(z)|^{q-2} u(z)+f(z, u(z)) \quad \text { in } \Omega, \\
\left.u\right|_{\partial \Omega}=0,1<q<p, \lambda>0 .
\end{array}\right.
$$

Here $\Delta_{p}$ denotes the $p$-Laplacian differential operator, defined by

$$
\Delta_{p} u=\operatorname{div}\left(\|\nabla u\|^{p-2} \nabla u\right) \quad \forall u \in W_{0}^{1, p}(\Omega),
$$

with $p \in(1,+\infty)$. In problem (1.1), the term $\lambda|u|^{q-2} u$ with $1<q<p$ is a $(p-1)$-sublinear term ("concave term"), while for the perturbation $f(z, \zeta)$, we assume that it is a Carathéodory function and for almost all $z \in \Omega$, the function $\zeta \longmapsto f(z, \zeta)$ exhibits a $(p-1)$-linear growth near $\pm \infty$. So, problem (1.1)

* The research was supported by the Marie Curie International Research Staff Exchange Scheme Fellowship within the 7th European Community Framework Programme under Grant Agreement No. 295118, the National Science Center of Poland under grant no. N N201 604640, the International Project co-financed by the Ministry of Science and Higher Education of Republic of Poland under grant no. W111/7.PR/2012 and the National Science Center of Poland under Maestro Advanced Project no. DEC-2012/06/A/ST1/00262.

Received 29 July 2011, in final form 4 May 2012. 
is asymptotically ( $p-1)$-linear. Our goal is to prove the existence of multiple nontrivial solutions for problem (1.1) and provide precise sign information for all of them.

In the past, problems with nonlinearities involving concave terms were studied primarily in the context of semilinear equations (i.e., $p=2$ ). We mention the works of Perera [24], de Paiva-Massa [4] and Wu-Yang [28], who have an asymptotically linear perturbation of the concave term. On the other hand, Ambrosetti-Brezis-Cerami [1] and Li-Wu-Zhou [19] consider equations with a right hand side nonlinearity of the form

$$
\lambda|u|^{q-2} u+|u|^{r-2} u,
$$

with $1<q<2<r<2^{*}$, where

$$
2^{*}= \begin{cases}\frac{2 N}{N-2} & \text { if } N \geqslant 3, \\ +\infty & \text { if } N=1,2 .\end{cases}
$$

So, in this case we have the combined effects of concave and convex nonlinearities. Extensions of their work to problems driven by the $p$-Laplacian, can be found in the works of Garcia Azorero-Manfredi-Peral Alonso [8], Guo-Zhang [16], Filippakis-Kristaly-Papageorgiou [7] and Kyritsi-Papageorgiou [17]. We should also mention the work of Wang [27], who has an odd perturbation of the concave term without any growth restriction near $\pm \infty$. Finally, we should also mention the recent works of Gasiński-Papageorgiou [11], [12] and of Papageorgiou-Rocha [23]. They also deal with problems in which the reaction $f(z, \cdot)$ is asymptotically $(p-1)$-linear at $\pm \infty$ (note that [11] considers Neumann problem). However, their conditions near the origin are different and do not allow for the presence of concave terms. In fact, in their setting $u=0$ is a local minimizer of the energy functional and this changes completely the geometry of the problem near the origin.

In this paper, we show that there exists a critical value $\lambda^{*}>0$, such that for all $\lambda \in\left(0, \lambda^{*}\right)$ problem (1.1) has at least five nontrivial smooth solutions, four of which have constant sign (two positive and two negative) and the fifth is nodal. Moreover, we show that, if $\lambda=\lambda^{*}$, then problem (1.1) still has three nontrivial smooth solutions, two of constant sign (one positive and the other negative) and the third is nodal. Hence, we could say that the solutions of problem (1.1) as functions of the parameter $\lambda>0$, exhibit a bifurcation-type behaviour for small values of $\lambda>0$ (the term "bifurcation" is used only in a very loose and suggestive way). In the semilinear case (i.e., $p=2$ ), we produce additional solutions. 
Our approach combines variational methods based on the critical point theory with Morse theory (critical groups) and suitable truncation techniques.

In the next Section, for the convenience of the reader, we briefly review the main mathematical tools that we will use in this paper.

\section{Mathematical Background}

In the analysis of problem (1.1), we will use the Sobolev space $W_{0}^{1, p}(\Omega)$ and the Banach space

$$
C_{0}^{1}(\bar{\Omega})=\left\{u \in C^{1}(\bar{\Omega}):\left.u\right|_{\partial \Omega}=0\right\} .
$$

The latter is an ordered Banach space with positive cone

$$
C_{+}=\left\{u \in C_{0}^{1}(\bar{\Omega}): u(z) \geqslant 0 \text { for all } z \in \bar{\Omega}\right\} .
$$

This cone has a nonempty interior, given by

$$
\text { int } C_{+}=\left\{u \in C_{+}: u(z)>0 \text { for all } z \in \Omega \text { and } \frac{\partial u}{\partial n}(z)<0 \text { for all } z \in \partial \Omega\right\} \text {. }
$$

Here $n(\cdot)$ denotes the outward unit normal on $\partial \Omega$.

Throughout this work, for every $u \in W_{0}^{1, p}(\Omega)$, we set $\|u\|=\|\nabla u\|_{p}$ and for every $\zeta \in \mathrm{R}$, we set $\zeta^{ \pm}=\max \{ \pm \zeta, 0\}$. The notation $\|\cdot\|$ will also be used to denote the $\mathrm{R}^{N}$-norm, but this will not create any confusion, since it will always be clear from the context, which one we are using. Finally by $|\cdot|_{N}$ we denote the Lebesgue measure on $\mathrm{R}^{N}$.

Let $X$ be a Banach space and let $X^{*}$ be its topological dual. By $\langle\cdot, \cdot\rangle$ we denote the duality brackets for the pair $\left(X^{*}, X\right)$. Let $\varphi \in C^{1}(X)$. A point $x_{0} \in X$ is called a critical point of $\varphi$, if $\varphi^{\prime}\left(x_{0}\right)=0$. A value $c \in \mathrm{R}$ is said to be a critical value of $\varphi$, if there exists a critical point $x_{0} \in X$, such that $\varphi\left(x_{0}\right)=c$. We introduce the following sets:

$$
\begin{aligned}
\varphi^{c} & =\{x \in X: \varphi(x) \leqslant c\}, \\
K^{\varphi} & =\left\{x \in X: \varphi^{\prime}(x)=0\right\}, \\
K_{c}^{\varphi} & =\left\{x \in K^{\varphi}: \varphi(x)=c\right\} .
\end{aligned}
$$

The following compactness-type condition plays a central role in critical point theory.

Definition 2.1. We say that $\varphi \in C^{1}(X)$ satisfies the Palais-Smale condition at level $c \in \mathrm{R}$ (the $P S_{c^{-}}$-condition for short), if every sequence $\left\{x_{n}\right\}_{n \geqslant 1} \subseteq X$, such that

$$
\varphi\left(x_{n}\right) \longrightarrow c \text { and } \varphi^{\prime}\left(x_{n}\right) \longrightarrow 0 \quad \text { in } X^{*},
$$


has a strongly convergent subsequence. We say that $\varphi \in C^{1}(X)$ satisfies the Palais-Smale condition (the $P S$-condition for short), if it satisfies the $P S_{c^{-}}$ condition at every level $c \in \mathrm{R}$.

Using this notion, we can have the following theorem known in the literature as the "mountain pass theorem".

Theorem 2.2. If $X$ is a Banach space, $\varphi \in C^{1}(X), x_{0}, x_{1} \in X, r>0$, $\left\|x_{1}-x_{0}\right\|>r$,

$$
\begin{aligned}
& \max \left\{\varphi\left(x_{0}\right), \varphi\left(x_{1}\right)\right\}<\inf \left\{\varphi(x):\left\|x-x_{0}\right\|=r\right\}=\eta_{r}, \\
& c=\inf _{\gamma \in \Gamma} \max _{0 \leqslant t \leqslant 1} \varphi(\gamma(t)),
\end{aligned}
$$

where

$$
\Gamma=\left\{\gamma \in C([0,1] ; X): \gamma(0)=x_{0}, \gamma(1)=x_{1}\right\}
$$

and $\varphi$ satisfies the $P S_{c}$-condition, then $c \geqslant \eta_{r}$ and $K_{c}^{\varphi} \neq \emptyset$.

The following notion from nonlinear operator theory, will be useful in proving that the energy functional of the problem satisfies the $P S$-condition.

Definition 2.3. A map $A: X \longrightarrow X^{*}$ is said to be "of type $(S)_{+}$", if for every sequence $\left\{x_{n}\right\}_{n \geqslant 1} \subseteq X$, such that

$$
x_{n} \longrightarrow x \quad \text { weakly in } X \quad \text { and } \quad \limsup _{n \rightarrow+\infty}\left\langle A\left(x_{n}\right), x_{n}-x\right\rangle \leqslant 0,
$$

one has

$$
x_{n} \longrightarrow x \quad \text { in } X \text {. }
$$

Next let us consider the map $A: W_{0}^{1, p}(\Omega) \longrightarrow W_{0}^{1, p}(\Omega)^{*}=W^{-1, p^{\prime}}(\Omega)$ corresponding to the $p$-Laplacian and defined by

$$
\langle A(u), y\rangle=\int_{\Omega}\|\nabla u\|^{p-2}(\nabla u, \nabla y)_{\mathrm{R}^{N}} d z \quad \forall u, y \in W_{0}^{1, p}(\Omega) .
$$

Then for this map, we have the following result (see Gasiński-Papageorgiou [9]).

Proposition 2.4. The map $A: W_{0}^{1, p}(\Omega) \longrightarrow W^{-1, p^{\prime}}(\Omega)$ defined by (2.1) is continuous, strictly monotone (hence maximal monotone too) and of type $(S)_{+}$.

Let $\left(Y_{1}, Y_{2}\right)$ be a topological pair with $Y_{2} \subseteq Y_{1} \subseteq X$. For every integer $k \geqslant 0$, by $H_{k}\left(Y_{1}, Y_{2}\right)$ we denote the $k$-th relative singular homology group for the pair $\left(Y_{1}, Y_{2}\right)$ with integer coefficients. 
Definition 2.5. The critical groups of $\varphi$ at an isolated critical point $x_{0} \in X$ with $c=\varphi\left(x_{0}\right)$ are defined by

$$
C_{k}\left(\varphi, x_{0}\right)=H_{k}\left(\varphi^{c} \cap U, \varphi^{c} \cap U \backslash\left\{x_{0}\right\}\right) \quad \forall k \geqslant 0,
$$

where $U$ is a neighbourhood of $x_{0}$, such that $K^{\varphi} \cap \varphi^{c} \cap U=\left\{x_{0}\right\}$.

The excision property of singular homology, implies that the above definition of critical groups is independent of the particular choice of the neighbourhood $U$.

Suppose that $\varphi \in C^{1}(X)$ satisfies the $P S$-condition and $-\infty<\inf \varphi\left(K^{\varphi}\right)$. We choose $c<\inf \varphi\left(K^{\varphi}\right)$.

DEFINITION 2.6. The critical groups of $\varphi$ at infinity are defined by

$$
C_{k}(\varphi, \infty)=H_{k}\left(X, \varphi^{c}\right) \quad \forall k \geqslant 0 .
$$

The second deformation theorem implies that the above definition is independent of the particular choice of the level $c<\inf \varphi\left(K^{\varphi}\right)$.

Suppose that $K^{\varphi}$ is finite and let us set

$$
M(t, x)=\sum_{k \geqslant 0} \operatorname{rank} C_{k}(\varphi, x) t^{k} \quad \forall x \in K^{\varphi}
$$

and

$$
P(t, \infty)=\sum_{k \geqslant 0} \operatorname{rank} C_{k}(\varphi, \infty) t^{k} .
$$

Then the Morse relation says that there is a polynomial $Q(t)$ with nonnegative integer coefficients, such that

$$
\sum_{x \in K^{\varphi}} M(t, x)=P(t, \infty)+(1+t) Q(t) .
$$

Finally let us recall some basic facts about the spectrum of the negative Dirichlet $p$-Laplacian, denoted by $-\Delta_{p}^{D}$. So, we consider the following nonlinear weighted eigenvalue problem with weight $m \in L^{\infty}(\Omega)_{+}, m \neq 0$ :

$$
\left\{\begin{array}{l}
-\Delta_{p} u(z)=\hat{\lambda} m(z)|u(z)|^{p-2} u(z) \quad \text { in } \Omega, \\
\left.u\right|_{\partial \Omega}=0 .
\end{array}\right.
$$

Every $\hat{\lambda} \in \mathrm{R}$ for which problem (2.3) has a nontrivial solution $u$, is said to be an eigenvalue of $-\Delta_{p}^{D}$ and $u$ is a corresponding eigenfunction. The smallest eigenvalue is denoted by $\hat{\lambda}_{1}(m)$ and it is positive, isolated (i.e., there 
exists $\varepsilon>0$ such that the interval $\left[\hat{\lambda}_{1}(m), \hat{\lambda}_{1}(m)+\varepsilon\right)$ contains no other eigenvalues), simple (i.e., the corresponding set of eigenfunctions is a onedimensional space) and it admits the following variational characterization (Rayleigh quotient):

$$
\hat{\lambda}_{1}(m)=\inf \left\{\frac{\|\nabla u\|_{p}^{p}}{\int_{\Omega} m|u|^{p} d z}: u \in W_{0}^{1, p}(\Omega), u \neq 0\right\} .
$$

In (2.4) the infimum is attained on the corresponding one-dimensional eigenspace. By $\hat{u}_{1}$ we denote the $L^{p}$-normalized (i.e., with $\left\|\hat{u}_{1}\right\|_{p}=1$ ) eigenfunction associated with $\hat{\lambda}_{1}(m)>0$. It is clear from (2.4), that $\hat{u}_{1}$ does not change sign and so we may assume that $\hat{u}_{1} \geqslant 0$. Nonlinear regularity theory (see e.g., Gasiński-Papageorgiou [9, pp. 737-738]) implies that $\hat{u}_{1} \in C_{+}$. In fact, the nonlinear maximum principle of Vázquez [26] implies that $\hat{u}_{1} \in \operatorname{int} C_{+}$.

Since the $p$-Laplacian is $(p-1)$-homogeneous map, the Ljusternik-Schnirelmann theory provides an increasing sequence $\left\{\hat{\lambda}_{k}(m)\right\}_{n \geqslant 1}$ of eigenvalues of $-\Delta_{p}^{D}$, known as the $L S$-eigenvalues of $-\Delta_{p}^{D}$, such that $\hat{\lambda}_{k}(m) \longrightarrow+\infty$ as $k \rightarrow+\infty$. If $p=2$ (linear eigenvalue problem), then these are all the eigenvalues of $-\Delta_{p}^{D}$. If $p \neq 2$ (nonlinear eigenvalue problem), we do not know if this is the case. Let $\sigma(p, m)$ denote the set of eigenvalues of (2.3). It is easily seen that $\sigma(p, m)$ is closed. Because $\hat{\lambda}_{1}(m)>0$ is isolated, we can define

$$
\hat{\lambda}_{2}^{*}(m)=\inf \left\{\hat{\lambda}: \hat{\lambda} \in \sigma(p, m), \hat{\lambda}>\hat{\lambda}_{1}(m)\right\}>\hat{\lambda}_{1}(m) .
$$

Evidently $\hat{\lambda}_{2}^{*}(m)$ is the second eigenvalue of $-\Delta_{p}^{D}$ and we have

$$
\hat{\lambda}_{2}^{*}(m)=\hat{\lambda}_{2}(m),
$$

i.e., for $-\Delta_{p}^{D}$ the second eigenvalue and the second $L S$-eigenvalue coincide. The function

$$
\left(L^{\infty}(\Omega)_{+} \backslash\{0\}\right) \ni m \longmapsto \hat{\lambda}_{k}(m) \in \mathbf{R}_{+} \backslash\{0\}
$$

is continuous and exhibits the following monotonicity properties:

- If $m(z) \leqslant m^{\prime}(z)$ for almost all $z \in \Omega$ and $m \neq m^{\prime}$, then $\hat{\lambda}_{1}\left(m^{\prime}\right)<\hat{\lambda}_{1}(m)$.

- If $m(z)<m^{\prime}(z)$ for almost all $z \in \Omega$, then $\hat{\lambda}_{2}\left(m^{\prime}\right)<\hat{\lambda}_{2}(m)$.

The following simple lemma can be found in Filippakis-Gasiński-Papageorgiou [6, Lemma 3.2] (see also Gasiński-Papageorgiou [13, Lemma 2.1]).

Lemma 2.7. If $\vartheta \in L^{\infty}(\Omega)_{+}, \vartheta(z) \leqslant \lambda_{1}$ for almost all $z \in \Omega$ and $\vartheta \neq \lambda_{1}$, then there exists $\xi_{0}>0$, such that

$$
\|\nabla u\|_{p}^{p}-\int_{\Omega} \vartheta|u|^{p} d z \geqslant \xi_{0}\|\nabla u\|_{p}^{p} \quad \forall u \in W_{0}^{1, p}(\Omega) .
$$


We will also need the notions of upper and lower solutions for problem (1.1).

DEFINITION 2.8.

(a) We say that $\bar{u} \in W^{1, p}(\Omega)$ is an upper solution for problem (1.1), if

$$
\left\{\begin{array}{lr}
\int_{\Omega}\|\nabla \bar{u}\|^{p-2}(\nabla \bar{u}, \nabla h)_{\mathrm{R}^{N}} d z \geqslant \lambda \int_{\Omega}|\bar{u}|^{q-2} \bar{u} h d z+\int_{\Omega} f(z, \bar{u}) h d z \\
\quad \forall h \in W_{0}^{1, p}(\Omega), h \geqslant 0, \\
\left.\bar{u}\right|_{\partial \Omega} \geqslant 0 .
\end{array}\right.
$$

We say that $\bar{u}$ is a strict upper solution, if it is an upper solution for (1.1) but not a solution.

(b) We say that $\underline{u} \in W_{0}^{1, p}(\Omega)$ is a lower solution for problem (1.1), if

$$
\left\{\begin{array}{lr}
\int_{\Omega}\|\nabla \underline{u}\|^{p-2}(\nabla \underline{u}, \nabla h)_{\mathrm{R}^{N}} d z \leqslant \lambda \int_{\Omega}|\bar{u}|^{q-2} \bar{u} h d z+\int_{\Omega} f(z, \underline{u}) h d z \\
\quad \forall h \in W_{0}^{1, p}(\Omega), h \geqslant 0, \\
\left.\underline{u}\right|_{\partial \Omega} \leqslant 0 . &
\end{array}\right.
$$

We say that $\underline{u}$ is a strict lower solution, if it is a lower solution for (1.1) but not a solution.

Finally recall that, if $S \subseteq W^{1, p}(\Omega)$, then we say that $S$ is downward directed (respectively upward directed), if for any $u_{1}, u_{2} \in S$, we can find $u_{3} \in S$, such that $u_{3} \leqslant \min \left\{u_{1}, u_{2}\right\}$ (respectively $u_{3} \geqslant \max \left\{u_{1}, u_{2}\right\}$ ).

\section{Solutions of Constant Sign}

The hypotheses on the nonlinearity $f(z, \zeta)$ are the following:

$H(f)_{1} \quad f: \Omega \times \mathrm{R} \longrightarrow \mathrm{R}$ is a function, such that:

(i) for all $\zeta \in \mathrm{R}$, the function $z \longmapsto f(z, \zeta)$ is measurable;

(ii) for almost all $z \in \Omega$, the function $\zeta \longmapsto f(z, \zeta)$ is continuous, $f(z, 0)=$ 0 ;

(iii) for every $r>0$, there exists $a_{r} \in L^{\infty}(\Omega)_{+}$, such that

$$
|f(z, \zeta)| \leqslant a_{r}(z) \quad \text { for almost all } z \in \Omega \text { and all }|\zeta| \leqslant r
$$

(iv) there exist functions $\eta_{1}, \eta_{2} \in L^{\infty}(\Omega)_{+}$, such that

$$
\eta_{1}(z) \geqslant \lambda_{1}, \quad \eta_{2}(z)<\lambda_{2} \quad \text { for almost all } z \in \Omega
$$


and $\eta_{1} \neq \lambda_{1}$ and

$$
\eta_{1}(z) \leqslant \liminf _{|\zeta| \rightarrow+\infty} \frac{f(z, \zeta)}{|\zeta|^{p-2} \zeta} \leqslant \limsup _{|\zeta| \rightarrow+\infty} \frac{f(z, \zeta)}{|\zeta|^{p-2} \zeta} \leqslant \eta_{2}(z)
$$

uniformly for almost all $z \in \Omega$;

(v) there exists $\vartheta \in L^{\infty}(\Omega)_{+}$, such that $\vartheta(z) \leqslant \lambda_{1}$ for almost all $z \in \Omega$, $\vartheta \neq \lambda_{1}$ and

$$
\lim _{\zeta \rightarrow 0} \frac{p F(z, \zeta)}{|\zeta|^{p}} \leqslant \vartheta(z)
$$

uniformly for almost all $z \in \Omega$, where

$$
F(z, \zeta)=\int_{0}^{\zeta} f(z, s) d s
$$

and

$$
f(z, \zeta) \zeta \geqslant-c_{0}|\zeta|^{p} \quad \text { for almost all } z \in \Omega \text {, all } \zeta \in \mathrm{R}
$$

with $c_{0}>0$.

REMARK 3.1. Hypothesis $H(f)_{1}$ (iv) dictates a $(p-1)$-linear growth at $\pm \infty$ in the spectral interval $\left[\lambda_{1}, \lambda_{2}\right]$. So, problem $(1.1)$ is asymptotically $(p-1)$ linear at infinity.

ExAMPLE 3.2. The following function $f(\zeta)$ satisfies hypotheses $H(f)_{1}$ (for the sake of simplicity we drop the $z$-dependence):

$$
f(\zeta)= \begin{cases}\vartheta|\zeta|^{r-2} \zeta & \text { if }|\zeta| \leqslant 1 \\ \eta|\zeta|^{p-2} \zeta+(\vartheta-\eta) \operatorname{sgn}(\zeta) & \text { if }|\zeta|>1\end{cases}
$$

with $0<\vartheta<\lambda_{1}<\eta<\lambda_{2}, p \leqslant r$.

As we already mentioned in the Introduction, our approach involves also truncation techniques. For this reason we introduce the following truncations of the reaction term in (1.1):

$$
\begin{aligned}
& f_{+}^{\lambda}(z, \zeta)= \begin{cases}0 & \text { if } \zeta \leqslant 0, \\
\lambda \zeta^{q-1}+f(z, \zeta) & \text { if } \zeta>0,\end{cases} \\
& f_{-}^{\lambda}(z, \zeta)= \begin{cases}\lambda|\zeta|^{q-2} \zeta+f(z, \zeta) & \text { if } \zeta<0, \\
0 & \text { if } \zeta \geqslant 0 .\end{cases}
\end{aligned}
$$

Both are Carathéodory functions. We set

$$
F_{ \pm}^{\lambda}(z, \zeta)=\int_{0}^{\zeta} f_{ \pm}^{\lambda}(z, s) d s
$$


and introduce the $C^{1}$-functionals $\varphi_{ \pm}^{\lambda}: W_{0}^{1, p}(\Omega) \longrightarrow \mathrm{R}$, defined by

$$
\varphi_{ \pm}^{\lambda}(u)=\frac{1}{p}\|\nabla u\|_{p}^{p}-\int_{\Omega} F_{ \pm}^{\lambda}(z, u(z)) d z \quad \forall u \in W_{0}^{1, p}(\Omega) .
$$

Also, we consider the $C^{1}$-energy functional $\varphi^{\lambda}: W_{0}^{1, p}(\Omega) \longrightarrow \mathrm{R}$ for problem (1.1), defined by

$$
\varphi^{\lambda}(u)=\frac{1}{p}\|\nabla u\|_{p}^{p}-\frac{\lambda}{p}\|u\|_{q}^{q}-\int_{\Omega} F(z, u(z)) d z \quad \forall u \in W_{0}^{1, p}(\Omega) .
$$

We show that the functionals $\varphi_{ \pm}^{\lambda}$ and $\varphi^{\lambda}$ satisfy the $P S$-condition.

Proposition 3.3. If hypotheses $H(f)_{1}$ hold and $\lambda>0$, then the functionals $\varphi_{ \pm}^{\lambda}, \varphi^{\lambda}: W_{0}^{1, p}(\Omega) \longrightarrow \mathrm{R}$ satisfy the PS-condition.

Proof. We do the proof for $\varphi_{+}^{\lambda}$, the proofs for $\varphi_{-}^{\lambda}$ and $\varphi^{\lambda}$ being similar.

So, let $\left\{u_{n}\right\}_{n \geqslant 1} \subseteq W_{0}^{1, p}(\Omega)$ be a sequence, such that $\left\{\varphi_{+}^{\lambda}\left(u_{n}\right)\right\} \subseteq \mathrm{R}$ is bounded and

$$
\left(\varphi_{+}^{\lambda}\right)^{\prime}\left(u_{n}\right) \longrightarrow 0 \quad \text { in } W^{-1, p^{\prime}}(\Omega)
$$

so

$$
\left|\left\langle A\left(u_{n}\right), h\right\rangle-\int_{\Omega} f_{+}^{\lambda}\left(z, u_{n}\right) h d z\right| \leqslant \varepsilon_{n}\|h\| \quad \forall h \in W_{0}^{1, p}(\Omega),
$$

with $\varepsilon_{n} \searrow 0$. In (3.5), we choose $h=-u_{n}^{-} \in W_{0}^{1, p}(\Omega)$. Then

$$
\left\|\nabla u_{n}^{-}\right\|_{p}^{p} \leqslant \varepsilon_{n}\left\|u_{n}^{-}\right\|
$$

and so

$$
u_{n}^{-} \longrightarrow 0 \quad \text { in } W_{0}^{1, p}(\Omega) .
$$

Suppose that $\left\|u_{n}\right\| \longrightarrow+\infty$. Then, from (3.6), we have that $\left\|u_{n}^{+}\right\| \longrightarrow+\infty$. We set

$$
y_{n}=\frac{u_{n}^{+}}{\left\|u_{n}^{+}\right\|} \quad \forall n \geqslant 1 .
$$

So, $\left\|y_{n}\right\|=1$ for all $n \geqslant 1$ and so passing to a suitable subsequence if necessary, we may assume that

$$
\begin{array}{ll}
y_{n} \longrightarrow y & \text { weakly in } W_{0}^{1, p}(\Omega), \\
y_{n} \longrightarrow y & \text { in } L^{p}(\Omega) .
\end{array}
$$


From (3.5), we have

$$
\begin{aligned}
\mid\left\langle A\left(y_{n}\right), h\right\rangle-\left\langle\frac{A\left(-u_{n}^{-}\right)}{\left\|u_{n}^{+}\right\|^{p-1}}, h\right\rangle-\int_{\Omega} \frac{f_{+}^{\lambda}\left(z, u_{n}^{+}\right)}{\left\|u_{n}^{+}\right\|^{p-1}} h d z \\
\quad-\int_{\Omega} \frac{f_{+}^{\lambda}\left(z,-u_{n}^{-}\right)}{\left\|u_{n}^{+}\right\|^{p-1}} h d z \mid \leqslant \varepsilon_{n} \frac{\|h\|}{\left\|u_{n}^{+}\right\|^{p-1}} \quad \forall h \in W_{0}^{1, p}(\Omega) .
\end{aligned}
$$

By virtue of (3.6), we have

$$
\begin{aligned}
\frac{A\left(-u_{n}^{-}\right)}{\left\|u_{n}^{+}\right\|^{p-1}} \longrightarrow 0 & \text { in } W^{-1, p^{\prime}}(\Omega) \\
\frac{f_{+}^{\lambda}\left(\cdot,-u_{n}^{-}(\cdot)\right)}{\left\|u_{n}^{+}\right\|^{p-1}} \longrightarrow 0 & \text { in } L^{p^{\prime}}(\Omega) .
\end{aligned}
$$

Therefore

$$
\left|\left\langle A\left(y_{n}\right), h\right\rangle-\int_{\Omega} \frac{f_{+}^{\lambda}\left(z, u_{n}^{+}\right)}{\left\|u_{n}^{+}\right\|^{p-1}} h d z\right| \leqslant \varepsilon_{n}^{\prime} \frac{\|h\|}{\left\|u_{n}^{+}\right\|^{p-1}} \quad \forall h \in W_{0}^{1, p}(\Omega),
$$

with $\varepsilon_{n}^{\prime} \searrow 0$. Note that

$$
u_{n}^{+}(z) \longrightarrow+\infty \quad \text { for almost all } z \in\{y>0\} .
$$

Using hypothesis $H(f)_{1}$ (iv) and reasoning as in Motreanu-Motreanu-Papageorgiou [22] (proof of Proposition 5), we can show that

$$
\frac{f_{+}^{\lambda}\left(\cdot, u_{n}^{+}(\cdot)\right)}{\left\|u_{n}^{+}\right\|^{p-1}} \longrightarrow g y^{p-1} \quad \text { weakly in } L^{p^{\prime}}(\Omega)
$$

with $g \in L^{\infty}(\Omega)_{+}, \eta_{1} \leqslant g \leqslant \eta_{2}$. In (3.9) we choose $h=y_{n}-y$ and then pass to the limit as $n \rightarrow+\infty$. Using (3.10), we obtain

$$
\lim _{n \rightarrow+\infty}\left\langle A\left(y_{n}\right), y_{n}-y\right\rangle=0 .
$$

From Proposition 2.4, we have that

$$
y_{n} \longrightarrow y \quad \text { in } W_{0}^{1, p}(\Omega)
$$

and so $\|y\|=1$. Therefore, if in (3.9) we pass to the limit as $n \rightarrow+\infty$ and use (3.10), then

$$
\langle A(y), h\rangle=\int_{\Omega} g y^{p-1} h d z \quad \forall h \in W_{0}^{1, p}(\Omega),
$$


so

$$
A(y)=g y^{p-1}
$$

and so

$$
\left\{\begin{array}{l}
-\Delta_{p} y(z)=g(z) y(z)^{p-1} \quad \text { in } \Omega \\
\left.y\right|_{\partial \Omega}=0, y \geqslant 0, \quad y \neq 0 .
\end{array}\right.
$$

From the monotonicity properties of the principal eigenvalues, we have

$$
\hat{\lambda}_{1}(g) \leqslant \hat{\lambda}_{1}\left(\eta_{1}\right)<\hat{\lambda}_{1}\left(\lambda_{1}\right)=1,
$$

so $y$ must change sign, a contradiction to (3.11). This proves that the sequence $\left\{u_{n}^{+}\right\}_{n \geqslant 1} \subseteq W_{0}^{1, p}(\Omega)$ is bounded, hence the sequence $\left\{u_{n}\right\}_{n \geqslant 1} \subseteq W_{0}^{1, p}(\Omega)$ is bounded (see (3.6)). So, passing to a subsequence if necessary, we may assume that

$$
\begin{array}{ll}
u_{n} \longrightarrow u & \text { weakly in } W_{0}^{1, p}(\Omega), \\
u_{n} \longrightarrow u & \text { in } L^{p}(\Omega) .
\end{array}
$$

In (3.5) we choose $h=u_{n}-u$ and then pass to the limit as $n \rightarrow+\infty$. We obtain

$$
\lim _{n \rightarrow+\infty}\left\langle A\left(u_{n}\right), u_{n}-u\right\rangle=0 .
$$

Using Proposition 2.4, we have that

$$
u_{n} \longrightarrow u \quad \text { in } W_{0}^{1, p}(\Omega)
$$

and so $\varphi_{+}^{\lambda}$ satisfies the $P S$-condition.

Similarly, we show that $\varphi_{-}^{\lambda}$ and $\varphi^{\lambda}$ satisfy the $P S$-condition.

Proposition 3.4. If hypotheses $H(f)_{1}$ hold, then there exists $\lambda_{+}^{*}>0$, such that for all $\lambda \in\left(0, \lambda_{+}^{*}\right)$, we can find a number $\varrho_{\lambda}>0$ for which

$$
\inf _{\partial B_{\varrho_{\lambda}}} \varphi_{+}^{\lambda}=\eta_{+}^{\lambda}>0
$$

where $\partial B_{\varrho_{\lambda}}=\left\{u \in W_{0}^{1, p}:\|u\|=\varrho_{\lambda}\right\}$.

Proof. By virtue of hypotheses $H(f)_{1}$ (iii) and (iv), we have

$$
|F(z, \zeta)| \leqslant a(z)+c|\zeta|^{p} \quad \text { for almost all } z \in \Omega \text {, all } \zeta \in \mathrm{R},
$$

with $a \in L^{\infty}(\Omega)_{+}, c>0$. From (3.12) and hypothesis $H(f)_{1}(\mathrm{v})$, we see that for a given $\varepsilon>0$, we can find $c_{\varepsilon}>0$, such that

(3.13) $F(z, \zeta) \leqslant \frac{\vartheta(z)+\varepsilon}{p} \zeta^{p}+c_{\varepsilon} \zeta^{\tau} \quad$ for almost all $z \in \Omega$, all $\zeta \geqslant 0$ 
with $\tau>p$. Then for every $u \in W_{0}^{1, p}(\Omega)$, we have

$$
\begin{aligned}
\varphi_{+}^{\lambda}(u) & =\frac{1}{p}\|\nabla u\|_{p}^{p}-\int_{\Omega} F_{+}^{\lambda}(z, u(z)) d z \\
& \geqslant \frac{1}{p}\|\nabla u\|_{p}^{p}-\frac{\lambda}{q}\|u\|_{q}^{q}-\frac{1}{p} \int_{\Omega} \vartheta|u|^{p} d z-\frac{\varepsilon}{p}\|u\|^{p}-\hat{c}_{\varepsilon}\|u\|^{\tau},
\end{aligned}
$$

for some $\hat{c}_{\varepsilon}>0$. Invoking Lemma 2.7 and choosing $\varepsilon \in\left(0, \xi_{0}\right)$, we obtain

$$
\begin{aligned}
\varphi_{+}^{\lambda}(u) & \geqslant c_{1}\|u\|^{p}-\lambda c_{2}\|u\|^{q}-\hat{c}_{\varepsilon}\|u\|^{\tau} \\
& =\left(c_{1}-\lambda c_{2}\|u\|^{q-p}-\hat{c}_{\varepsilon}\|u\|^{\tau-p}\right)\|u\|^{p},
\end{aligned}
$$

for some $c_{1}, c_{2}>0$.

We consider the function

$$
\sigma_{\lambda}(t)=\lambda c_{2} t^{q-p}+\hat{c}_{\varepsilon} t^{\tau-p} \quad t>0 .
$$

Note that

$$
\begin{array}{ll}
\sigma_{\lambda}(t) \longrightarrow+\infty & \text { as } t \rightarrow+\infty \\
\sigma_{\lambda}(t) \longrightarrow+\infty & \text { as } t \rightarrow 0^{+}
\end{array}
$$

(since $q<p<\tau)$. Of course $\sigma_{\lambda}$ is continuous on $(0,+\infty)$. Therefore, we can find $t_{0}>0$, such that

$$
\sigma_{\lambda}\left(t_{0}\right)=\inf _{\mathrm{R}_{+}} \sigma_{\lambda}
$$

so

$$
\sigma_{\lambda}^{\prime}\left(t_{0}\right)=0
$$

and thus

$$
t_{0}=t_{0}(\lambda)=\left(\frac{\lambda c_{2}(p-q)}{\hat{c}_{\varepsilon}(\tau-p)}\right)^{\frac{1}{\tau-q}} .
$$

We consider $\sigma_{\lambda}\left(t_{0}\right)$ in (3.15) and we see that we can find $\lambda_{+}^{*}>0$, such that

$$
\sigma_{\lambda}\left(t_{0}\right)<c_{1} \quad \forall \lambda \in\left(0, \lambda_{+}^{*}\right) .
$$

So, from (3.14), it follows that

$$
\varphi_{+}^{\lambda}(u) \geqslant \eta_{\lambda}^{+}>0 \quad \forall\|u\|=\varrho_{\lambda}=t_{0}(\lambda), \lambda \in\left(0, \lambda_{+}^{*}\right) .
$$

In a similar fashion, we also show the following proposition. 
Proposition 3.5. If hypotheses $H(f)_{1}$ hold, then there exists $\lambda_{-}^{*}>0$, such that for all $\lambda \in\left(0, \lambda_{-}^{*}\right)$, we can find a number $\varrho_{\lambda}>0$ for which

$$
\inf _{\partial B_{e_{\lambda}}} \varphi_{-}^{\lambda}=\eta_{-}^{\lambda}>0
$$

Recall that $\hat{u}_{1} \in \operatorname{int} C_{+}$is the $L^{p}$-normalized principal eigenvalue of $-\Delta_{p}^{D}$.

Proposition 3.6. If hypotheses $H(f)_{1}$ hold and $\lambda>0$, then $\varphi_{ \pm}^{\lambda}\left(t \hat{u}_{1}\right) \longrightarrow$ $-\infty$ as $t \rightarrow \pm \infty$.

Proof. We do the proof for $\varphi_{+}^{\lambda}$, the proof for $\varphi_{-}^{\lambda}$ being similar.

By virtue of hypotheses $H(f)_{1}$ (iii) and (iv), for a given $\varepsilon>0$, we can find $\bar{c}_{\varepsilon}>0$, such that

(3.16) $F(z, \zeta) \geqslant \frac{\eta_{1}(z)-\varepsilon}{p}|\zeta|^{p}-\bar{c}_{\varepsilon} \quad$ for almost all $z \in \Omega$ and all $\zeta \in \mathrm{R}$.

Hence, using (3.16), for $t>0$, we have

$$
\begin{aligned}
\varphi_{+}^{\lambda}\left(t \hat{u}_{1}\right) & =\frac{t^{p}}{p}\left\|\nabla \hat{u}_{1}\right\|_{p}^{p}-\int_{\Omega} F_{+}^{\lambda}\left(z, t \hat{u}_{+}\right) d z \\
& \leqslant \frac{t^{p}}{p}\left(\int_{\Omega}\left(\lambda_{1}-\eta_{1}(z)\right) \hat{u}_{1}(z)^{p} d z+\varepsilon\right)+\bar{c}_{\varepsilon}|\Omega|_{N} .
\end{aligned}
$$

From the hypothesis on $\eta_{1}$ (see $H(f)_{1}($ iv) $)$ and since $\hat{u}_{1} \in \operatorname{int} C_{+}$, we have

$$
\xi_{1}=\int_{\Omega}\left(\lambda_{1}-\eta_{1}(z)\right) \hat{u}_{1}(z)^{p} d z<0 .
$$

We choose $\varepsilon \in\left(0,-\xi_{1}\right)$. Then from (3.17), it follows that

$$
\varphi_{+}^{\lambda}\left(t \hat{u}_{1}\right) \longrightarrow-\infty \quad \text { as } t \rightarrow+\infty
$$

In a similar fashion, we show that

$$
\varphi_{+}^{\lambda}\left(t \hat{u}_{1}\right) \longrightarrow-\infty \quad \text { as } t \rightarrow-\infty .
$$

We are ready to produce the constant sign solutions for problem (1.1).

Proposition 3.7. If hypotheses $H(f)_{1}$ hold and $\lambda_{ \pm}^{*}>0$ are as postulated in Propositions 3.4 and 3.5, then

(a) for all $\lambda \in\left(0, \lambda_{+}^{*}\right)$, problem (1.1) has a solution $u_{0} \in \operatorname{int} C_{+}$;

(b) for all $\lambda \in\left(0, \lambda_{-}^{*}\right)$, problem (1.1) has a solution $v_{0} \in-\operatorname{int} C_{+}$; 
(c) for all $\lambda \in\left(0, \lambda^{*}\right)$, where $\lambda^{*}=\min \left\{\lambda_{+}^{*}, \lambda_{-}^{*}\right\}$, problem (1.1) has two nontrivial smooth constant sign solutions: $u_{0} \in \operatorname{int} C_{+}$and $v_{0} \in-\operatorname{int} C_{+}$.

Proof. (a) Propositions 3.3, 3.4 and 3.6 permit the use of the mountain pass theorem (see Theorem 2.2). So, for $\lambda \in\left(0, \lambda_{+}^{*}\right)$ (see Proposition 3.4), we can find $u_{0} \in W_{0}^{1, p}(\Omega)$, such that

$$
\varphi_{+}^{\lambda}(0)=0<\eta_{+}^{\lambda} \leqslant \varphi_{+}^{\lambda}\left(u_{0}\right)
$$

and

$$
\left(\varphi_{+}^{\lambda}\right)^{\prime}\left(u_{0}\right)=0 .
$$

From (3.18), it follows that $u_{0} \neq 0$. From (3.19), we have that

$$
A\left(u_{0}\right)=N_{+}^{\lambda}\left(u_{0}\right),
$$

where $N_{+}^{\lambda}(u)(\cdot)=f_{+}^{\lambda}(\cdot, u(\cdot))$ for all $u \in W_{0}^{1, p}(\Omega)$.

On (3.20), we act with $-u_{0}^{-} \in W_{0}^{1, p}(\Omega)$. Then

$$
\left\|\nabla u_{0}^{-}\right\|_{p}^{p}=0
$$

so $u_{0}^{-}=0$, i.e.,

$$
u_{0} \geqslant 0, u_{0} \neq 0 \text {. }
$$

Hence (3.20) becomes

$$
A\left(u_{0}\right)=\lambda u_{0}^{q-1}+N\left(u_{0}\right),
$$

where $N(u)(\cdot)=f(\cdot, u(\cdot))$ for all $u \in W_{0}^{1, p}(\Omega)$, so

$$
\left\{\begin{array}{l}
-\Delta_{p} u_{0}(z)=\lambda u_{0}(z)^{q-1}+f\left(z, u_{0}(z)\right) \quad \text { in } \Omega, \\
\left.u\right|_{\partial \Omega}=0 .
\end{array}\right.
$$

and using hypothesis $H(f)_{1}(\mathrm{v})$, we have

$$
\Delta_{p} u_{0}(z) \leqslant c_{0} u_{0}(z)^{p-1} \quad \text { for almost all } z \in \Omega .
$$

From (3.21) and the nonlinear regularity theory (see e.g., Gasiński-Papageorgiou [9, pp. 737-738]), we have $u_{0} \in C_{+}$, while from (3.22) and the nonlinear maximum principle of Vázquez [26], we have that $u_{0} \in \operatorname{int} C_{+}$.

(b) The proof is similar with that of (a), working this time with $\varphi_{-}^{\lambda}, \lambda \in$ $\left(0, \lambda_{-}^{*}\right)$ and using Proposition 3.5 (instead of Proposition 3.4). So, we obtain a solution $v_{0} \in-\operatorname{int} C_{+}$for problem (1.1), for $\lambda \in\left(0, \lambda_{-}^{*}\right)$. 
(c) This part follows by combining (a) and (b).

Proposition 3.8. If hypotheses $H(f)_{1}$ hold and $\lambda \in\left(0, \lambda_{+}^{*}\right)$ (respectively $\left.\lambda \in\left(0, \lambda_{-}^{*}\right)\right)$, then $\inf _{\bar{B}_{e_{\lambda}}} \varphi_{+}^{\lambda}<0$ (respectively $\left.\inf _{\bar{B}_{e_{\lambda}}} \varphi_{-}^{\lambda}<0\right)$.

Proof. We do the proof for $\varphi_{+}^{\lambda}$, the proof for $\varphi_{-}^{\lambda}$ being similar.

Let $t>0$ be small, such that $t\left\|\hat{u}_{1}\right\|<\varrho_{\lambda}$. Then, using hypothesis $H(f)_{1}(\mathrm{v})$ and recalling that $\left\|\hat{u}_{1}\right\|_{p}=1$, for $\lambda \in\left(0, \lambda_{+}^{*}\right)$, we have

$$
\begin{aligned}
\varphi_{+}^{\lambda}\left(t \hat{u}_{1}\right) & =\frac{t^{p}}{p}\left\|\nabla \hat{u}_{1}\right\|_{p}^{p}-\int_{\Omega} F_{+}^{\lambda}\left(z, t \hat{u}_{1}\right) d z \\
& \leqslant t^{q}\left(\frac{\lambda_{1}+c_{0}}{p} t^{p-q}-\frac{\lambda}{r}\left\|\hat{u}_{1}\right\|_{q}^{q}\right) .
\end{aligned}
$$

Since $q<p$, by choosing $t>0$ even smaller if necessary, we have

$$
\frac{\lambda_{1}+c_{0}}{p} t^{p-q}-\frac{\lambda}{r}\left\|\hat{u}_{1}\right\|_{q}^{q}<0,
$$

so

$$
\varphi_{+}^{\lambda}\left(t \hat{u}_{1}\right)<0
$$

and thus

$$
\inf _{\bar{B}_{e_{\lambda}}} \varphi_{+}^{\lambda}<0
$$

Similarly for $\varphi_{-}^{\lambda}$, with $\lambda \in\left(0, \lambda_{-}^{*}\right)$.

Next we use Proposition 3.8 and the Ekeland variational principle (see e.g., Gasiński-Papageorgiou [9, p. 582]), in order to produce two more nontrivial smooth solutions of constant sign.

Proposition 3.9. If hypotheses $H(f)_{1}$ hold, then

(a) for all $\lambda \in\left(0, \lambda_{+}^{*}\right)$, problem (1.1) has two nontrivial positive smooth solutions $u_{0}, \hat{u} \in \operatorname{int} C_{+}$and $\hat{u}$ is a local minimizer of $\varphi^{\lambda}$;

(b) for all $\lambda \in\left(0, \lambda_{-}^{*}\right)$, problem (1.1) has two nontrivial negative smooth solutions $v_{0}, \hat{v} \in-$ int $C_{+}$and $\hat{v}$ is a local minimizer of $\varphi^{\lambda}$;

(c) for all $\lambda \in\left(0, \lambda^{*}\right)$, where $\lambda^{*}=\min \left\{\lambda_{+}^{*}, \lambda_{-}^{*}\right\}$, problem (1.1) has four nontrivial smooth constant sign solutions: $u_{0}, \hat{u} \in \operatorname{int} C_{+}$and $v_{0}, \hat{v} \in$ - int $C_{+}$.

Proof. (a) Let

$$
\beta=\inf _{\partial B_{Q_{\lambda}}} \varphi_{+}^{\lambda}-\inf _{\bar{B}_{\varrho_{\lambda}}} \varphi_{+}^{\lambda}>0
$$


(see Propositions 3.4 and 3.8). Then for $\varepsilon \in(0, \beta)$, we apply the Ekeland variational principle (see e.g., Gasiński-Papageorgiou [9, p. 582]) and obtain $u_{\varepsilon} \in \bar{B}_{Q_{\lambda}}$, such that

$$
\varphi_{+}^{\lambda}\left(u_{\varepsilon}\right) \leqslant \inf _{\bar{B}_{e_{\lambda}}} \varphi_{+}^{\lambda}+\varepsilon
$$

and

$$
\varphi_{+}^{\lambda}\left(u_{\varepsilon}\right) \leqslant \varphi_{+}^{\lambda}(u)+\varepsilon\left\|u-u_{\varepsilon}\right\| \quad \forall u \in \bar{B}_{\varrho_{\lambda}} .
$$

From (3.23) and the choice of $\varepsilon \in(0, \beta)$, we have

$$
\varphi_{+}^{\lambda}\left(u_{\varepsilon}\right)<\inf _{\partial B_{Q_{\lambda}}} \varphi_{+}^{\lambda},
$$

so $u_{\varepsilon} \in \bar{B}_{\varrho_{\lambda}} \backslash \partial B_{Q_{\lambda}}$.

Let $h \in W_{0}^{1, p}(\Omega)$. Since $u_{\varepsilon} \in B_{\varrho_{\lambda}}$, for $t>0$ small, we have $u_{\varepsilon}+t h \in B_{\varrho_{\lambda}}$. Substituting in (3.24), dividing by $t$ and letting $t \rightarrow 0$, we obtain

$$
\left\langle\left(\varphi_{+}^{\lambda}\right)^{\prime}\left(u_{\varepsilon}\right), h\right\rangle \geqslant-\varepsilon\|h\| \quad \forall h \in W_{0}^{1, p}(\Omega)
$$

and thus

$$
\left\|\left(\varphi_{+}^{\lambda}\right)^{\prime}\left(u_{\varepsilon}\right)\right\|_{*} \leqslant \varepsilon .
$$

Let $\varepsilon_{n}=\frac{1}{n}$ and $u_{n}=u_{\varepsilon_{n}}$. Then from (3.23) and (3.25), it follows that

$$
\varphi_{+}^{\lambda}\left(u_{n}\right) \longrightarrow \inf _{\bar{B}_{e_{\lambda}}} \varphi_{+}^{\lambda}
$$

and

$$
\left(\varphi_{+}^{\lambda}\right)^{\prime}\left(u_{n}\right) \longrightarrow 0 \quad \text { in } W_{0}^{1, p}(\Omega) .
$$

From (3.26), (3.27), Proposition 3.3 and by passing to a subsequence if necessary, we may assume that

$$
u_{n} \longrightarrow \hat{u} \quad \text { in } W_{0}^{1, p}(\Omega) .
$$

Using (3.26) and Propositions 3.4 and 3.8, we obtain

$$
\varphi_{+}^{\lambda}\left(u_{n}\right) \longrightarrow \varphi_{+}^{\lambda}(\hat{u})=\inf _{\bar{B}_{e_{\lambda}}} \varphi_{+}^{\lambda}<0=\varphi_{+}^{\lambda}(0)<\inf _{\partial B_{e_{\lambda}}} \varphi_{+}^{\lambda} .
$$

Therefore $\hat{u} \in \bar{B}_{Q_{\lambda}} \backslash \partial B_{Q_{\lambda}}, \hat{u} \neq 0$ and we can say that

$$
\left(\varphi_{+}^{\lambda}\right)^{\prime}(\hat{u})=0,
$$


so

$$
A(\hat{u})=N_{+}^{\lambda}(\hat{u})
$$

Acting with $-\hat{u}^{-} \in W_{0}^{1, p}(\Omega)$, we obtain $\hat{u} \geqslant 0, \hat{u} \neq 0$. Hence

$$
A(\hat{u})=\lambda \hat{u}^{q-1}+N(\hat{u}),
$$

so

$$
\left\{\begin{array}{l}
-\Delta_{p} \hat{u}(z)=\lambda \hat{u}(z)^{q-1}+f(z, \hat{u}(z)) \quad \text { in } \Omega \\
\left.\hat{u}\right|_{\partial \Omega}=0
\end{array}\right.
$$

Therefore $\hat{u} \in C_{+}$(nonlinear regularity theory) and by the nonlinear maximum principle of Vázquez [26] (see hypothesis $H(f)_{1}(\mathrm{v})$ ), we have that $\hat{u} \in \operatorname{int} C_{+}$.

Since

$$
\left.\varphi_{+}^{\lambda}\right|_{C_{+}}=\left.\varphi^{\lambda}\right|_{C_{+}}
$$

we see that $\hat{u} \in$ int $C_{+}$is a local $C_{0}^{1}(\bar{\Omega})$-minimizer of $\varphi^{\lambda}$. Invoking Theorem 1.2 of Garcia Azorero-Manfredi-Peral Alonso [8], we infer that $\hat{u}$ is a local $W_{0}^{1, p}(\Omega)$-minimizer of $\varphi^{\lambda}$.

(b) The proof of this part is similar to that of (a), using this time the functional $\varphi_{-}^{\lambda}$.

(c) This part is obtained by combining (a) and (b).

Next we show that for fixed $\lambda \in\left(0, \lambda_{+}^{*}\right)$ (respectively $\left.\lambda \in\left(0, \lambda_{-}^{*}\right)\right)$ problem (1.1) has a smallest positive solution (respectively biggest negative solution). To do this, we shall need the following lemma which can be found in GasińskiPapageorgiou [10].

LEMMA 3.10.

(a) The set of upper solutions for problem (1.1) is downward directed. More precisely for any two upper solutions $y_{1}, y_{2} \in W^{1, p}(\Omega)$, the function $y=\min \left\{y_{1}, y_{2}\right\} \in W^{1, p}(\Omega)$ is an upper solution too.

(b) The set of lower solutions for problem (1.1) is upward directed. More precisely for any two lower solutions $v_{1}, v_{2} \in W^{1, p}(\Omega), v=\max \left\{v_{1}, v_{2}\right\} \in$ $W^{1, p}(\Omega)$ is a lower solution too.

Using this lemma, we can produce extremal constant sign solutions for problem (1.1). For this, we will need the following stronger version of hypotheses $H(f)_{1}$.

$$
\underline{H(f)_{2}} \quad f: \Omega \times \mathrm{R} \longrightarrow \mathrm{R} \text { is a function, such that: }
$$

(i)-(iv) the same as hypotheses $H(f)_{1}$ (i)-(iv); 
(v) there exists $\vartheta \in L^{\infty}(\Omega)_{+}$, such that $\vartheta(z) \leqslant \lambda_{1}$ for almost all $z \in \Omega$, $\vartheta \neq \lambda_{1}$ and

$$
\lim _{\zeta \rightarrow+\infty} \frac{p F(z, \zeta)}{|\zeta|^{p}} \leqslant \vartheta(z),
$$

uniformly for almost all $z \in \Omega$ and

$$
f(z, \zeta) \zeta \geqslant 0 \quad \text { for almost all } z \in \Omega, \text { all } \zeta \in \mathrm{R} .
$$

REMARK 3.11. The function given in Example 3.2 still satisfies hypotheses $H(f)_{2}$.

Proposition 3.12. If hypotheses $H(f)_{2}$ hold and $\lambda \in\left(0, \lambda^{*}\right)$, where $\lambda^{*}=$ $\min \left\{\lambda_{+}^{*}, \lambda_{-}^{*}\right\}$, then problem (1.1) has a smallest positive solution $u_{+} \in \operatorname{int} C_{+}$ and a biggest negative solution $v_{-} \in-$ int $C_{+}$.

Proof. Choose $\varepsilon \in(0,1)$ small, such that

$$
\lambda_{1} \varepsilon^{p-1} \hat{u}_{1}(z)^{p-1}<\lambda \varepsilon^{q-1} \hat{u}_{1}(z)^{q-1} \quad \forall z \in \Omega
$$

(recall $q<p$ ). We set $\underline{u}=\varepsilon \hat{u}_{1} \in$ int $C_{+}$. Then, using (3.28) and hypothesis $H(f)_{2}(v)$, we have

$$
\begin{aligned}
-\Delta_{p} \underline{u}(z) & =-\Delta_{p}\left(\varepsilon \hat{u}_{1}\right)(z)=\lambda_{1} \varepsilon^{p-1} \hat{u}_{1}(z)^{p-1} \\
& <\lambda \varepsilon^{q-1} \hat{u}_{1}(z)^{q-1} \leqslant \lambda \underline{u}(z)^{q-1}+f(z, \underline{u}(z))
\end{aligned}
$$

for almost all $z \in \Omega$, so $\underline{u} \in \operatorname{int} C_{+}$is a lower solution for problem (1.1).

Let $\underline{S}_{+}$be the set of solutions $u$ of problem (1.1), such that $\underline{u} \leqslant u$. Вy choosing $\varepsilon \in(0,1)$ small, such that $\varepsilon \hat{u}_{1} \leqslant \min \left\{u_{0}, \hat{u}\right\}$, we see that $\underline{S}_{+} \neq \emptyset$.

We will show that $\underline{S}_{+}$is downward directed. To this end, let $u_{1}, u_{2} \in$ $\underline{S}_{+}$. Evidently both are upper solutions for problem (1.1) and so according to Lemma $3.10(\mathrm{a}), \bar{u}=\min \left\{u_{1}, u_{2}\right\} \in W_{0}^{1, p}(\Omega)$ is an upper solution too and of course $\underline{u} \leqslant \bar{u}$. We set

$$
\hat{f}_{\lambda}(z, \zeta)= \begin{cases}\lambda \underline{u}(z)^{q-1}+f(z, \underline{u}(z)) & \text { if } \zeta<\underline{u}(z), \\ \lambda \zeta^{q-1}+f(z, \zeta) & \text { if } \underline{u}(z) \leqslant \zeta \leqslant \bar{u}(z), \\ \lambda \bar{u}(z)^{q-1}+f(z, \bar{u}(z)) & \text { if } \bar{u}(z)<\zeta\end{cases}
$$

Evidently $\hat{f}_{\lambda}(z, \zeta)$ is a Carathéodory function. Let

$$
\hat{F}_{\lambda}(z, \zeta)=\int_{0}^{\zeta} \hat{f}_{\lambda}(z, s) d s
$$


We consider the $C^{1}$-functional $\hat{\varphi}_{\lambda}: W_{0}^{1, p}(\Omega) \longrightarrow \mathrm{R}$, defined by

$$
\hat{\varphi}_{\lambda}(u)=\frac{1}{p}\|\nabla u\|_{p}^{p}-\int_{\Omega} \hat{F}_{\lambda}(z, u(z)) d z \quad \forall u \in W_{0}^{1, p}(\Omega) .
$$

Because of (3.29), we have

$$
\hat{\varphi}_{\lambda}(u) \geqslant \frac{1}{p}\|\nabla u\|_{p}^{p}-c_{3} \quad \forall u \in W_{0}^{1, p}(\Omega),
$$

for some $c_{3}>0$, so the functional $\hat{\varphi}_{\lambda}$ is coercive.

In addition, exploiting the compactness of the embedding $W_{0}^{1, p}(\Omega) \subseteq$ $L^{p}(\Omega)$, we can easily verify that the functional $\hat{\varphi}_{\lambda}$ is sequentially weakly lower semicontinuous. So, by the Weierstrass theorem, we can find $\hat{u}_{0} \in W_{0}^{1, p}(\Omega)$, such that

$$
\hat{\varphi}_{\lambda}\left(\hat{u}_{0}\right)=\inf _{u \in W_{0}^{1, p}(\Omega)} \hat{\varphi}_{\lambda}(u),
$$

So

$$
\hat{\varphi}_{\lambda}^{\prime}\left(\hat{u}_{0}\right)=0
$$

and thus

$$
A\left(\hat{u}_{0}\right)=\hat{N}_{\lambda}\left(\hat{u}_{0}\right),
$$

where

$$
\hat{N}_{\lambda}(u)(\cdot)=\hat{f}_{\lambda}(\cdot, u(\cdot)) \quad \forall u \in W_{0}^{1, p}(\Omega) .
$$

On (3.30), we act with $\left(\underline{u}-\hat{u}_{0}\right)^{+} \in W_{0}^{1, p}(\Omega)$. Then, using (3.29) and the fact that $\underline{u} \in \operatorname{int} C_{+}$is a lower solution of (1.1), we have

$$
\begin{aligned}
\left\langle A\left(\hat{u}_{0}\right),\left(\underline{u}-\hat{u}_{0}\right)^{+}\right\rangle= & \int_{\left\{\underline{u}>u_{0}\right\}} \hat{f}_{\lambda}\left(z, \hat{u}_{0}\right)\left(\underline{u}-\hat{u}_{0}\right) d z \\
= & \lambda \int_{\left\{\underline{u}>u_{0}\right\}} \underline{u}^{q-1}\left(\underline{u}-\hat{u}_{0}\right) d z \\
& \quad+\int_{\left\{\underline{u}>u_{0}\right\}} f(z, \underline{u})\left(\underline{u}-\hat{u}_{0}\right) d z \\
\geqslant & \left\langle A(\underline{u}),\left(\underline{u}-\hat{u}_{0}\right)^{+}\right\rangle,
\end{aligned}
$$

so

$$
\int_{\left\{\underline{u}>u_{0}\right\}}\left(\left\|\nabla \hat{u}_{0}\right\|^{p-2} \nabla \hat{u}_{0}-\|\nabla \underline{u}\|^{p-2} \nabla \underline{u}, \nabla \underline{u}-\nabla \hat{u}_{0}\right)_{\mathrm{R}^{N}} \geqslant 0,
$$

thus $\left|\left\{\underline{u}>\hat{u}_{0}\right\}\right|_{N}=0$. So, finally

$$
\underline{u} \leqslant \hat{u}_{0} .
$$


Similarly, acting on (3.30) with $\left(\hat{u}_{0}-\bar{u}\right)^{+} \in W_{0}^{1, p}(\Omega)$, we show that $\hat{u}_{0} \leqslant \bar{u}$. So, from (3.29) and (3.30), it follows that

$$
\left\{\begin{array}{l}
-\Delta_{p} \hat{u}_{0}(z)=\lambda \hat{u}_{0}(z)^{q-1}+f\left(z, \hat{u}_{0}(z)\right) \quad \text { in } \Omega \\
\left.\hat{u}_{0}\right|_{\partial \Omega}=0
\end{array}\right.
$$

so

$$
\hat{u}_{0} \in \underline{S}_{+} .
$$

This proves that the set $\underline{S}_{+}$is downward directed.

We consider a chain $C \subseteq \underline{S}_{+}$(i.e., the set $C$ is totally ordered). From Dunford-Schwartz [5, Corollary 7, p. 336], we know that there exists a sequence $\left\{u_{n}\right\}_{n \geqslant 1} \subseteq C$, such that

$$
\inf _{n \geqslant 1} u_{n}=\inf C .
$$

In fact, since $C$ is totally ordered, we may assume that the sequence $\left\{u_{n}\right\}_{n \geqslant 1}$ is decreasing. We have

$$
A\left(u_{n}\right)=\lambda u_{n}^{q-1}+N\left(u_{n}\right) \quad \forall n \geqslant 1 .
$$

Recalling that $0 \leqslant u_{n} \leqslant u_{1}$ for $n \geqslant 1$, we have

$$
\left\|\nabla u_{n}\right\|_{p}^{p} \leqslant c_{4}\left\|u_{1}\right\|_{q}^{q} \quad \forall n \geqslant 1
$$

for $c_{4}>0$ and so the sequence $\left\{u_{n}\right\}_{n \geqslant 1} \subseteq W_{0}^{1, p}(\Omega)$ is bounded.

Hence we may assume that

$$
\begin{array}{ll}
u_{n} \longrightarrow u & \text { weakly in } W_{0}^{1, p}(\Omega), \\
u_{n} \longrightarrow u & \text { in } L^{p}(\Omega) .
\end{array}
$$

On (3.31) we act with $u_{n}-u \in W_{0}^{1, p}(\Omega)$ and pass to the limit as $n \rightarrow+\infty$. We obtain

$$
\lim _{n \rightarrow+\infty}\left\langle A\left(u_{n}\right), u_{n}-u\right\rangle=0,
$$

so using Proposition 2.4, we have

$$
u_{n} \longrightarrow u \quad \text { in } W_{0}^{1, p}(\Omega) .
$$

So, if we pass to the limit in (3.31) as $n \rightarrow+\infty$, we obtain

$$
A(u)=\lambda u^{q-1}+N(u), \quad \underline{u} \leqslant u,
$$


so

$$
\left\{\begin{array}{l}
-\Delta_{p} u(z)=\lambda u(z)^{q-1}+f(z, u(z)) \quad \text { in } \Omega \\
\left.u\right|_{\partial \Omega}=0, \underline{u} \leqslant u
\end{array}\right.
$$

thus

$$
u \in \underline{S}_{+} \quad \text { and } \quad u=\inf C .
$$

Invoking the Kuratowski-Zorn lemma, we infer that the set $\underline{S}_{+}$has a minimal element $x_{*} \in \underline{S}_{+}$. But recall that $\underline{S}_{+}$is downward directed. Therefore we conclude that $u_{*} \geqslant \underline{u}$ must be the smallest solution of (1.1) in

$$
I_{+}=\left\{u \in W_{0}^{1, p}(\Omega): \underline{u} \leqslant u\right\} .
$$

Now let $\varepsilon_{n} \searrow 0$ and let us set

$$
\underline{u}_{n}=\varepsilon_{n} \hat{u}_{1}
$$

and

$$
I_{+}^{n}=\left\{u \in W_{0}^{1, p}(\Omega): \underline{u}_{n} \leqslant u\right\} .
$$

From the above argument, we know that problem (1.1) has a smallest solution $u_{*}^{n}$ in $I_{+}^{*}$. Evidently, the sequence $\left\{u_{*}^{n}\right\}_{n \geqslant 1} \subseteq W_{0}^{1, p}(\Omega)$ is bounded and so we may assume that

$$
\begin{array}{ll}
u_{*}^{n} \longrightarrow u_{+} & \text {weakly in } W_{0}^{1, p}(\Omega), \\
u_{*}^{n} \longrightarrow u_{+} & \text {in } L^{p}(\Omega) .
\end{array}
$$

We have

$$
A\left(u_{*}^{n}\right)=\lambda\left(u_{*}^{n}\right)^{q-1}+N\left(u_{*}^{n}\right) \quad \forall n \geqslant 1 .
$$

On (3.32) we act with $u_{*}^{n}-u_{+}$and then pass to the limit as $n \rightarrow+\infty$. We obtain

$$
\lim _{n \rightarrow+\infty}\left\langle A\left(u_{*}^{n}\right), u_{*}^{n}-u_{+}\right\rangle=0 .
$$

Using Proposition 2.4, we have

$$
u_{*}^{n} \longrightarrow u_{+} \quad \text { in } W_{0}^{1, p}(\Omega) .
$$

So, if we pass to the limit as $n \rightarrow+\infty$, and using (3.32), we obtain

$$
A\left(u_{+}\right)=\lambda\left(u_{+}\right)^{q-1}+N\left(u_{+}\right)
$$

so

$$
\left\{\begin{array}{l}
-\Delta_{p} u_{+}(z)=\lambda u_{+}(z)^{q-1}+f\left(z, u_{+}(z)\right) \quad \text { in } \Omega \\
\left.u_{+}\right|_{\partial \Omega}=0
\end{array}\right.
$$


thus $u_{+} \in C_{+}$(by the nonlinear regularity theory) is a solution of problem (1.1).

Next we show that $u_{+} \in \operatorname{int} C_{+}$. To this end we consider the following auxiliary Dirichlet problem:

$$
\left\{\begin{array}{l}
-\Delta_{p} u(z)=\lambda u(z)^{q-1} \quad \text { in } \Omega, \\
\left.u\right|_{\partial \Omega}=0, u \geqslant 0 .
\end{array}\right.
$$

The Euler functional for problem (3.33) is of the form

$$
\psi_{\lambda}(u)=\frac{1}{p}\|\nabla u\|_{p}^{p}-\frac{\lambda}{q}\left\|u^{+}\right\|_{q}^{q} \quad \forall u \in W_{0}^{1, p}(\Omega) .
$$

Since $q<p, \psi_{\lambda}$ is coercive and of course sequentially weakly lower semicontinuous. So, we can find $\underline{u}^{\lambda} \in W_{0}^{1, p}(\Omega)$, such that

$$
\psi_{\lambda}\left(\underline{u}^{\lambda}\right)=\inf _{u \in W_{0}^{1, p}(\Omega)} \psi_{\lambda}(u) .
$$

Note that since $q<p$, for $\varepsilon \in(0,1)$ small enough, we have

$$
\psi_{\lambda}\left(\varepsilon \hat{u}_{1}\right)<0=\psi_{\lambda}(0),
$$

hence from (3.34), it follows that $\underline{u}^{\lambda} \neq 0$ and

$$
\left(\psi_{\lambda}\right)^{\prime}\left(\underline{u}^{\lambda}\right)=0 \text {, }
$$

so

$$
A\left(\underline{u}^{\lambda}\right)=\lambda\left(\left(\underline{u}^{\lambda}\right)^{+}\right)^{q-1} .
$$

Acting on (3.35) with $-\left(\underline{u}^{\lambda}\right)^{-}$, we obtain

$$
\underline{u}^{\lambda} \geqslant 0, \quad \underline{u}^{\lambda} \neq 0 .
$$

Also, the nonlinear regularity theory and nonlinear maximum principle of Vázquez [26] implies that $\underline{u}_{\lambda} \in \operatorname{int} C_{+}$. Since $u_{*}^{n} \in$ int $C_{+}$, we can find $\xi_{n}>0$, such that

$$
\xi_{n} \underline{u}^{\lambda} \leqslant u_{*}^{n} \quad \forall n \geqslant 1 .
$$

We take $\xi_{n}>0$ to be the biggest number for which (3.36) holds. Suppose that $\xi_{n} \in(0,1)$. Then, using hypothesis $H(f)_{2}(\mathrm{v}),(3.36)$ and (3.33), we have

$$
\begin{aligned}
-\Delta_{p} u_{*}^{n}(z) & \geqslant \lambda u_{*}^{n}(z)^{q-1} \geqslant \lambda\left(\xi_{n} \underline{u}^{\lambda}(z)\right)^{q-1} \\
& >\lambda \xi_{n}^{p-1} \underline{u}^{\lambda}(z)^{q-1}=-\Delta_{p}\left(\xi_{n} \underline{u}^{\lambda}\right)(z)
\end{aligned}
$$

for almost all $z \in \Omega$. 
Invoking Proposition 2.2 of Guedda-Veron [15], we have

$$
u_{*}^{n}-\xi_{n} \underline{u}^{\lambda} \in \operatorname{int} C_{+},
$$

which contradicts the maximality of $\xi_{n}>0$. Therefore $\xi_{n} \geqslant 1$ for all $n \geqslant 1$ and so

$$
\underline{u}^{\lambda} \leqslant u_{*}^{n} \quad \forall n \geqslant 1,
$$

so

$$
\underline{u}^{\lambda} \leqslant u_{+}, \quad \text { i.e., } u_{+} \in \text { int } C_{+} \text {. }
$$

We claim that $u_{+}$is the smallest positive solution of (1.1). To this end, let $u \in W_{0}^{1, p}(\Omega)$ be a positive solution of (1.1). Nonlinear regularity and the maximum principle of Vázquez [26], imply that $u \in \operatorname{int} C_{+}$. Hence for all $n \geqslant 1$ large enough, we have

$$
\underline{u}_{n}=\varepsilon_{n} \hat{u}_{1} \leqslant u,
$$

so

$$
u_{*}^{n} \leqslant u
$$

and thus $u_{+} \leqslant u$. So, indeed $u_{+} \in$ int $C_{+}$is the smallest positive solution of problem (1.1).

Similarly, using this time Lemma 3.10 (b), we can produce the biggest negative solution $v_{-} \in-$ int $C_{+}$of problem (1.1) and $v_{-} \leqslant \bar{v}^{\lambda}$, where $\bar{v}^{\lambda} \in$ - int $C_{+}$is a solution of the following auxiliary Dirichlet problem:

$$
\left\{\begin{array}{l}
-\Delta_{p} v(z)=\lambda|v(z)|^{p-2} v(z) \quad \text { in } \Omega, \\
\left.v\right|_{\partial \Omega}=0, v \leqslant 0 .
\end{array}\right.
$$

A solution $\bar{v}^{\lambda} \in-$ int $C_{+}$of (3.38) can be obtained using the direct method on the Euler functional

$$
\hat{\psi}_{\lambda}(v)=\frac{1}{p}\|\nabla v\|_{p}^{p}-\frac{\lambda}{q}\left\|v^{-}\right\|_{q}^{q} \quad \forall v \in W_{0}^{1, p}(\Omega)
$$

of problem (3.38) (see also problem (3.33) earlier in the proof).

\section{Nodal Solutions}

In this section, using Proposition 3.12, we prove the full multiplicity theorem for problem (1.1), which produces a fifth nontrivial smooth solution for problem (1.1), which is nodal (see Proposition 3.9 (c)). We need the following stronger hypotheses on $f(z, \zeta)$ :

$$
\underline{H(f)_{3}} \quad f: \Omega \times \mathrm{R} \longrightarrow \mathrm{R} \text { is a function, such that: }
$$


(i)-(v) the same as hypotheses $H(f)_{2}(\mathrm{i})-(\mathrm{v})$;

(vi) there exist $\mu, r, \hat{c}_{0}>0$, such that $q \leqslant \mu<p<r$ and

$$
\mu F(z, \zeta)-f(z, \zeta) \zeta \geqslant-\hat{c}_{0}|\zeta|^{r} \quad \text { for almost all } z \in \Omega \text { and all } \zeta \in \mathrm{R} \text {. }
$$

REMARK 4.1. The function $f$ given in Example 3.2 still satisfies hypotheses $H(f)_{3}$ provided that $r>p$.

First we compute the critical groups of $\varphi^{\lambda}$ at $u=0$. Our proof uses some ideas of Moroz [21].

Proposition 4.2. If hypotheses $H(f)_{3}$ hold and $\lambda>0$, then

$$
C_{k}\left(\varphi^{\lambda}, 0\right)=0 \quad \forall k \geqslant 0 .
$$

Proof. Let

$$
f_{\lambda}(z, \zeta)=\lambda|\zeta|^{q-2} \zeta+f(z, \zeta)
$$

and

$$
F_{\lambda}(z, \zeta)=\int_{0}^{\zeta} f_{\lambda}(z, s) d s
$$

Because of the sign condition in hypothesis $H(f)_{3}(\mathrm{v})$, we have

$$
F_{\lambda}(z, \zeta) \geqslant \frac{\lambda}{q}|\zeta|^{q} \quad \text { for almost all } z \in \Omega, \text { all } \zeta \in \mathrm{R} .
$$

If $u \in W_{0}^{1, p}(\Omega), u \neq 0$ and $t>0$, then

$$
\begin{aligned}
\varphi^{\lambda}(t u) & =\frac{t^{p}}{p}\|\nabla u\|_{p}^{p}-\int_{\Omega} F_{\lambda}(z, t u) d z \\
& \leqslant \frac{t^{p}}{p}\|\nabla u\|_{p}^{p}-\frac{\lambda t^{q}}{q}\|u\|_{q}^{q} .
\end{aligned}
$$

Because $q<p$, it is clear from (4.1) that we can find $t^{*}=t^{*}(u) \in(0,1)$, such that

$$
\varphi^{\lambda}(t u)<0 \quad \forall t \in\left(0, t^{*}\right)
$$

Let $u \in W_{0}^{1, p}(\Omega), u \neq 0$ be such that $\varphi^{\lambda}(u)=0$. Then

$$
-\frac{\mu}{p}\|\nabla u\|_{p}^{p}+\frac{\lambda \mu}{q}\|u\|_{q}^{q}+\int_{\Omega} \mu F(z, u) d z=0 .
$$


Also, using (4.3), hypothesis $H(f)_{2}$ (vi) and recalling that $\mu<p$, we have

$$
\begin{aligned}
\left.\frac{d}{d t} \varphi^{\lambda}(t u)\right|_{t=1}= & \left\langle\left(\varphi^{\lambda}\right)^{\prime}(u), u\right\rangle=\langle A(u), u\rangle-\int_{\Omega} f_{\lambda}(z, u) u d z \\
= & \left(1-\frac{\mu}{p}\right)\|\nabla u\|_{p}^{p}+\lambda\left(\frac{\mu}{q}-1\right)\|u\|_{q}^{q} \\
& \quad+\int_{\Omega}(\mu F(z, u)-f(z, u) u) d z \\
\geqslant & \left(1-\frac{\mu}{p}\right)\|\nabla u\|_{p}^{p}-\hat{c}_{0}\|u\|_{r}^{r} \\
\geqslant & c_{5}\|u\|^{p}-c_{6}\|u\|^{r},
\end{aligned}
$$

for some $c_{5}, c_{6}>0$. Because $r>p$ (see hypothesis $H(f)_{3}$ (vi)), from (4.4), we see that we can find $\varrho \in(0,1)$ small, such that

(4.5) $\left.\frac{d}{d t} \varphi^{\lambda}(t u)\right|_{t=1}>0 \quad \forall u \in W_{0}^{1, p}(\Omega)$, with $0<\|u\| \leqslant \varrho, \varphi^{\lambda}(u)=0$.

Now, let $u \in W_{0}^{1, p}(\Omega)$ with $0<\|u\| \leqslant \varrho$ and $\varphi^{\lambda}(u) \leqslant 0$. We will show that

$$
\varphi^{\lambda}(t u) \leqslant 0 \quad \forall t \in[0,1] .
$$

We argue by contradiction. So, suppose that we can find $t_{0} \in(0,1)$, such that $\varphi^{\lambda}\left(t_{0} u\right)>0$. Since $\varphi^{\lambda}(u) \leqslant 0$, by virtue of the continuity of $\varphi^{\lambda}(\cdot)$, we can find $t_{1} \in\left(t_{0}, 1\right]$, such that

$$
\varphi^{\lambda}\left(t_{1} u\right)=0 .
$$

Let $t_{1} \in\left[t_{0}, 1\right]$ be the smallest such number. Evidently $0<t_{0}<t_{1}$ and

$$
\varphi^{\lambda}(t u)>0 \quad \forall t \in\left[t_{0}, t_{1}\right) .
$$

Let us set $y=t_{1} u$. Then $0<\|y\| \leqslant\|u\| \leqslant \varrho$ and $\varphi^{\lambda}(y)=0$. Therefore, by virtue of (4.5), we have

$$
\left.\frac{d}{d t} \varphi^{\lambda}(t y)\right|_{t=1}>0
$$

On the other hand, from (4.7), we have

$$
\varphi^{\lambda}(y)=\varphi^{\lambda}\left(t_{1} u\right)=0<\varphi^{\lambda}(t u) \quad \forall t \in\left[t_{0}, t_{1}\right),
$$


so

$$
\left.\frac{d}{d t} \varphi^{\lambda}(t y)\right|_{t=1}=\left.t_{1} \frac{d}{d t} \varphi^{\lambda}(t u)\right|_{t=t_{1}}=t_{1} \lim _{t \rightarrow t_{1}^{-}} \frac{\varphi^{\lambda}(t u)}{t-t_{1}} \leqslant 0 .
$$

Combining (4.8) and (4.9) we reach a contradiction. This proves (4.6).

By taking $\varrho \in(0,1)$ even smaller if necessary, we may assume that $u=0$ is the only critical point of $\varphi^{\lambda}$ in $\bar{B}_{\varrho}$, otherwise we have a whole sequence of distinct nontrivial smooth solutions of (1.1) and so we are done (here $\bar{B}_{Q}=$ $\left.\left\{u \in W_{0}^{1, p}(\Omega):\|u\| \leqslant \varrho\right\}\right)$. Let $h:[0,1] \times\left(\bar{B}_{\varrho} \cap\left(\varphi^{\lambda}\right)^{0}\right) \longrightarrow \bar{B}_{\varrho} \cap\left(\varphi^{\lambda}\right)^{0}$ be defined by

$$
h(t, u)=(1-t) u .
$$

By virtue of (4.6), this map is well defined and continuous (hence a homotopy). It follows that $\bar{B}_{\varrho} \cap\left(\varphi^{\lambda}\right)^{0}$ is contractible in itself.

Suppose that $u \in \bar{B}_{\varrho}$ with $\varphi^{\lambda}(u)>0$. We will show that there exists a unique $t(u) \in(0,1)$, such that

$$
\varphi^{\lambda}(t(u) u)=0 .
$$

The existence of $t(u) \in(0,1)$ follows from (4.2), the continuity of $\varphi^{\lambda}(\cdot)$ and the fact that $\varphi^{\lambda}(u)>0$. We show the uniqueness of $t(u) \in(0,1)$. So, suppose that

$$
0<t_{1}(u)<t_{2}(u)<1 \quad \text { and } \quad \varphi^{\lambda}\left(t_{k}(u) u\right)=0 \quad \text { for } k=1,2 .
$$

From (4.6), we have

$$
\varphi^{\lambda}\left(t t_{2}(u) u\right) \leqslant 0 \quad \forall t \in[0,1] .
$$

Therefore, if we consider the function $t \longmapsto \varphi^{\lambda}\left(t t_{2}(u) u\right)$ on [0, 1], then this function achieves its maximum at $\frac{t_{1}(u)}{t_{2}(u)} \in(0,1)$ and so

$$
\left.\frac{d}{d t} \varphi^{\lambda}\left(t t_{1}(u) u\right)\right|_{t=1}=\left.\frac{t_{1}(u)}{t_{2}(u)} \frac{d}{d t} \varphi^{\lambda}\left(t t_{2}(u) u\right)\right|_{t=\frac{t_{1}(u)}{t_{2}(u)}}=0 .
$$

Comparing (4.11) and (4.5), we reach a contradiction (recall $\varphi^{\lambda}\left(t_{1}(u) u\right)=0$ ). This proves the uniqueness of $t(u) \in(0,1)$. Evidently, using (4.6) and (4.10), we have

$$
\begin{cases}\varphi^{\lambda}(t u)<0 & \forall t \in(0, t(u)) \\ \varphi^{\lambda}(t u)>0 & \forall t \in(t(u), 1] .\end{cases}
$$


Let $\xi: \bar{B}_{\varrho} \backslash\{0\} \longrightarrow(0,1]$ be defined by

$$
\xi(u)= \begin{cases}1 & \text { if } u \in \bar{B}_{\varrho} \backslash\{0\}, \varphi^{\lambda}(u) \leqslant 0, \\ t(u) & \text { if } u \in \bar{B}_{\varrho} \backslash\{0\}, \varphi^{\lambda}(u)>0 .\end{cases}
$$

We claim that $\xi$ is continuous. We need to check the continuity only at those $u$, such that $\varphi^{\lambda}(u)=0$, since at the other points the continuity follows from the implicit function theorem. So, suppose that

$$
u_{n} \longrightarrow u \text { and } \varphi^{\lambda}\left(u_{n}\right)>0 \quad \forall n \geqslant 1 .
$$

Arguing by contradiction, suppose that

$$
t\left(u_{n}\right) \leqslant t^{*}<1 \quad \forall n \geqslant 1 .
$$

Then, from (4.12), we have

$$
\varphi^{\lambda}\left(t u_{n}\right)>0 \quad \forall t \in\left(t^{*}, 1\right], n \geqslant 1,
$$

so

$$
\varphi^{\lambda}(t u) \geqslant 0 \quad \forall t \in\left(t^{*}, 1\right] .
$$

Using also (4.6) and recalling that $\varphi^{\lambda}(u)=0$, we have

$$
\varphi^{\lambda}(t u)=0 \quad \forall t \in\left(t^{*}, 1\right],
$$

so

$$
\left.\frac{d}{d t} \varphi^{\lambda}(t u)\right|_{t=1}=0
$$

which contradicts (4.5).

This proves the continuity of the map $\xi$.

Consider the map $\sigma: \bar{B}_{\varrho} \backslash\{0\} \longrightarrow\left(\bar{B}_{\varrho} \cap\left(\varphi^{\lambda}\right)^{0}\right) \backslash\{0\}$, defined by

$$
\sigma(u)=\xi(u) u \text {. }
$$

Then $\sigma$ is continuous and

$$
\left.\sigma\right|_{\left(\bar{B}_{e} \cap\left(\varphi^{\lambda}\right)^{0}\right) \backslash\{0\}}=\text { identity. }
$$

This proves that $\left(\bar{B}_{\varrho} \cap\left(\varphi^{\lambda}\right)^{0}\right) \backslash\{0\}$ is a retraction of $\bar{B}_{\varrho} \backslash\{0\}$ and the latter is contractible. Recall that a retract of a contractible set, is itself contractible. Therefore $\left(\bar{B}_{\varrho} \cap\left(\varphi^{\lambda}\right)^{0}\right) \backslash\{0\}$ is contractible and so from Granas-Dugundji [14, p. 389], we have

$$
C_{k}\left(\varphi^{\lambda}, 0\right)=H_{k}\left(\bar{B}_{\varrho} \cap\left(\varphi^{\lambda}\right)^{0},\left(\bar{B}_{\varrho} \cap\left(\varphi^{\lambda}\right)^{0}\right) \backslash\{0\}\right)=0 \quad \forall k \geqslant 0 .
$$


Theorem 4.3. If hypotheses $H(f)_{3}$ hold, $\lambda^{*}$ is as postulated in Proposition 3.7 and $\lambda \in\left(0, \lambda^{*}\right)$, then problem (1.1) has at least five nontrivial smooth solutions

$$
u_{0}, \hat{u} \in \operatorname{int} C_{+}, \quad v_{0}, \hat{v} \in-\operatorname{int} C_{+} \quad \text { and } \quad y_{0} \in C_{0}^{1}(\bar{\Omega}) \text { nodal. }
$$

Proof. From Proposition 3.9(c), we already have four nontrivial constant sign smooth solutions

$$
u_{0}, \hat{u} \in \operatorname{int} C_{+}, \quad v_{0}, \hat{v} \in-\operatorname{int} C_{+} .
$$

Let $u_{+} \in \operatorname{int} C_{+}$and $v_{-} \in-$ int $C_{+}$be the two extremal constant sign solutions of problem (1.1), obtained in Proposition 3.12. We introduce the following truncations of the nonlinearity $f(z, \zeta)$ :

$$
\begin{aligned}
& \hat{f}_{+}^{\lambda}(z, \zeta)= \begin{cases}0 & \text { if } \zeta<0, \\
\lambda \zeta^{q-1}+f(z, \zeta) & \text { if } 0 \leqslant \zeta \leqslant u_{+}(z), \\
\lambda u_{+}(z)^{q-1}+f\left(z, u_{+}(z)\right) & \text { if } u_{+}(z)<\zeta,\end{cases} \\
& \hat{f}_{-}^{\lambda}(z, \zeta)= \begin{cases}\lambda\left|v_{-}(z)\right|^{q-2} v_{-}(z)+f\left(z, v_{-}(z)\right) & \text { if } \zeta<v_{-}(z), \\
\lambda|\zeta|^{q-2} \zeta+f(z, \zeta) & \text { if } v_{-}(z) \leqslant \zeta \leqslant 0, \\
0 & \text { if } 0<\zeta,\end{cases} \\
& \hat{f}^{\lambda}(z, \zeta)= \begin{cases}\lambda\left|v_{-}(z)\right|^{q-2} v_{-}(z)+f\left(z, v_{-}(z)\right) & \text { if } \zeta<v_{-}(z), \\
\lambda|\zeta|^{q-2} \zeta+f(z, \zeta) & \text { if } v_{-}(z) \leqslant \zeta \leqslant u_{+}(z), \\
\lambda u_{+}(z)^{q-1}+f\left(z, u_{+}(z)\right) & \text { if } u_{+}(z)<\zeta .\end{cases}
\end{aligned}
$$

These are Carathéodory functions. We set

$$
\begin{aligned}
& \hat{F}_{ \pm}^{\lambda}(z, \zeta)=\int_{0}^{\zeta} \hat{f}_{ \pm}^{\lambda}(z, s) d s, \\
& \hat{F}^{\lambda}(z, \zeta)=\int_{0}^{\zeta} \hat{f}^{\lambda}(z, s) d s
\end{aligned}
$$

and introduce the $C^{1}$-functionals $\hat{\varphi}_{ \pm}^{\lambda}, \hat{\varphi}^{\lambda}: W_{0}^{1, p}(\Omega) \longrightarrow \mathrm{R}$, defined by

$$
\begin{array}{ll}
\hat{\varphi}_{ \pm}^{\lambda}(u)=\frac{1}{p}\|\nabla u\|_{p}^{p}-\int_{\Omega} \hat{F}_{ \pm}^{\lambda}(z, u(z)) d z & \forall u \in W_{0}^{1, p}(\Omega), \\
\hat{\varphi}^{\lambda}(u)=\frac{1}{p}\|\nabla u\|_{p}^{p}-\int_{\Omega} \hat{F}^{\lambda}(z, u(z)) d z & \forall u \in W_{0}^{1, p}(\Omega) .
\end{array}
$$


Claim 1. The critical points of $\hat{\varphi}_{+}^{\lambda}$ are $\left\{0, u_{+}\right\}$and the critical points of $\hat{\varphi}_{-}^{\lambda}$ are $\left\{v_{-}, 0\right\}$.

We give the proof for $\hat{\varphi}_{+}^{\lambda}$, the proof for $\hat{\varphi}_{-}^{\lambda}$ being similar.

So, suppose that $u \in W_{0}^{1, p}(\Omega)$ is a nontrivial critical point of $\hat{\varphi}_{+}^{\lambda}$. Then

$$
\left(\hat{\varphi}_{+}^{\lambda}\right)^{\prime}(u)=0,
$$

so

$$
A(u)=\hat{N}_{+}^{\lambda}(u),
$$

where $\hat{N}_{+}^{\lambda}(y)(\cdot)=\hat{f}_{+}^{\lambda}(\cdot, y(\cdot))$ for all $y \in W_{0}^{1, p}(\Omega)$.

On (4.13), we act with $\left(u-u_{+}\right)^{+} \in W_{0}^{1, p}(\Omega)$. Then

$$
\begin{aligned}
\left\langle A(u),\left(u-u_{+}\right)^{+}\right\rangle & =\int_{\left\{u>u_{+}\right\}} \hat{f}_{+}^{\lambda}(z, u)\left(u-u_{+}\right) d z \\
& =\int_{\Omega}\left(\lambda u_{+}^{q-1}+f\left(z, u_{+}\right)\right)\left(u-u_{+}\right)^{+} d z \\
& =\left\langle A\left(u_{+}\right),\left(u-u_{+}\right)^{+}\right\rangle,
\end{aligned}
$$

so

$$
\left\langle A(u)-A\left(u_{+}\right),\left(u-u_{+}\right)^{+}\right\rangle=0,
$$

thus

$$
\int_{\left\{u>u_{+}\right\}}\left(\|\nabla u\|^{p-2} \nabla u-\left\|\nabla u_{+}\right\|^{p-2} \nabla u_{+}, \nabla u-\nabla u_{+}\right)_{\mathrm{R}^{N}} d z=0,
$$

so $\left|\left\{u>u_{+}\right\}\right|_{N}=0$ and finally we get that $u \leqslant u_{+}$.

Also acting on (4.13) with $-u^{-} \in W_{0}^{1, p}(\Omega)$, we obtain

$$
\left\|\nabla u^{-}\right\|_{p}^{p}=0
$$

i.e., $u^{-}=0$, hence

$$
u \geqslant 0, \quad u \neq 0 .
$$

Due to the extremality of $u_{+}$, we must have $u=u_{+}$.

Similarly, we show that the critical points of $\hat{\varphi}_{-}^{\lambda}$ are $\left\{0, v_{-}\right\}$. This proves Claim 1.

Let

$$
\left[v_{-}, u_{+}\right]=\left\{u \in W_{0}^{1, p}(\Omega): v_{-}(z) \leqslant u(z) \leqslant u_{+}(z) \text { for almost all } z \in \Omega\right\} .
$$

Claim 2. The critical points of $\hat{\varphi}^{\lambda}$ are in $\left[v_{-}, u_{+}\right]$. 
Indeed, if $u \in W_{0}^{1, p}(\Omega)$ is a critical point of $\hat{\varphi}^{\lambda}$, then

$$
\left(\hat{\varphi}^{\lambda}\right)^{\prime}(u)=0
$$

so

$$
A(u)=\hat{N}^{\lambda}(u),
$$

where $\hat{N}^{\lambda}(y)(\cdot)=\hat{f}^{\lambda}(\cdot, y(\cdot))$ for all $y \in W_{0}^{1, p}(\Omega)$.

Acting on (4.14), first with $\left(u-u_{+}\right)^{+} \in W_{0}^{1, p}(\Omega)$ and then with $\left(v_{-}-u\right)^{+} \in$ $W_{0}^{1, p}(\Omega)$ as above, we obtain $u \in\left[v_{-}, u_{+}\right]$. This proves Claim 2.

Claim 3. $u_{+}$and $v_{-}$are local minimizers of the functional $\hat{\varphi}^{\lambda}$.

Choose $t \in(0,1)$ small, such that $t \hat{u}_{1} \leqslant u_{+}$(recall that $u_{+} \in \operatorname{int} C_{+}$). Then, from hypothesis $H(f)_{2}(\mathrm{v})$, for almost all $z \in \Omega$, we have

$$
\hat{F}_{+}^{\lambda}\left(z, t \hat{u}_{1}\right)=\frac{\lambda t^{q}}{q} \hat{u}_{1}(z)^{q}+F\left(z, t \hat{u}_{1}(z)\right) \geqslant \frac{\lambda t^{q}}{q} \hat{u}_{1}(z)^{q} .
$$

Therefore, from (4.15) and the fact that $\left\|\hat{u}_{1}\right\|_{p}=1$, we have

$$
\hat{\varphi}_{+}^{\lambda}\left(t \hat{u}_{1}\right) \leqslant \frac{t^{p}}{p} \lambda_{1}-\frac{\lambda t^{q}}{q}\left\|\hat{u}_{1}\right\|_{q}^{q} .
$$

Since $q<p$, by choosing $t \in(0,1)$ even smaller if necessary, we have

$$
\hat{\varphi}_{+}^{\lambda}\left(t \hat{u}_{1}\right)<0
$$

so

$$
\inf _{u \in W_{0}^{1, p}(\Omega)} \hat{\varphi}_{+}^{\lambda}(u)=\hat{m}_{+}^{\lambda}<0 .
$$

Clearly $\hat{\varphi}_{+}^{\lambda}$ is coercive and we can easily verify that it is sequentially weakly lower semicontinuous. So, applying the Weierstrass theorem, we can find $\tilde{u} \in$ $W_{0}^{1, p}(\Omega)$, such that

$$
\hat{\varphi}_{+}^{\lambda}(\tilde{u})=\hat{m}_{+}^{\lambda}<0=\hat{\varphi}_{+}^{\lambda}(0)
$$

(see (4.16)), so $\tilde{u} \neq 0$ and from Claim 1, we get that

$$
\tilde{u}=u_{+} .
$$

But $u_{+} \in \operatorname{int} C_{+}$(see Proposition 3.12). So, we can find $r>0$ small, such that

$$
\left.\hat{\varphi}_{+}^{\lambda}\right|_{\bar{B}_{r}^{C_{0}^{1}(\bar{\Omega})}\left(u_{+}\right)}=\left.\hat{\varphi}^{\lambda}\right|_{\bar{B}_{r}^{C_{0}^{1}(\bar{\Omega})}\left(u_{+}\right)},
$$


where

$$
\bar{B}^{C_{0}^{1}(\bar{\Omega})}\left(u_{+}\right)=\left\{u \in C_{0}^{1}(\bar{\Omega}):\left\|u-u_{+}\right\|_{C_{0}^{1}(\bar{\Omega})} \leqslant r\right\}
$$

Hence $u_{+}$is a local $C_{0}^{1}(\bar{\Omega})$-minimizer of $\hat{\varphi}^{\lambda}$ and by Theorem 1.2 of Garcia Azorero-Manfredi-Peral Alonso [8], we infer that $u_{+}$is a local $W_{0}^{1, p}(\Omega)$-minimizer of $\hat{\varphi}^{\lambda}$.

Similarly, we show that $v_{-} \in-$ int $C_{+}$is a local $W_{0}^{1, p}(\Omega)$-minimizer of $\hat{\varphi}^{\lambda}$. This proves Claim 3.

Exploiting the coercivity of $\hat{\varphi}^{\lambda}$, we can easily check that $\hat{\varphi}^{\lambda}$ satisfies the $P S$-condition. This fact together with Claim 1, permit the use of the mountain pass theorem (see Theorem 2.2). So, we obtain $y_{0} \in W_{0}^{1, p}(\Omega) \backslash\left\{v_{-}, u_{+}\right\}$, such that

$$
\left(\hat{\varphi}^{\lambda}\right)^{\prime}\left(y_{0}\right)=0
$$

so from Claim 2, we have

$$
y_{0} \in\left[v_{-}, u_{+}\right]
$$

Therefore $y_{0}$ solves problem (1.1) and $y_{0} \in C_{0}^{1}(\bar{\Omega})$ by the nonlinear regularity theory. Moreover, $y_{0}$ being a critical point of mountain pass type, we must have

$$
C_{1}\left(\hat{\varphi}^{\lambda}, y_{0}\right) \neq 0 \text {. }
$$

On the other hand, from Proposition 4.2, we have

$$
C_{k}\left(\hat{\varphi}^{\lambda}, 0\right)=0 \quad \forall k \geqslant 0 .
$$

Therefore $y_{0} \neq 0$. Since $y_{0} \in\left[v_{-}, u_{+}\right], y_{0} \notin\left\{0, v_{-}, u_{+}\right\}$, by virtue of the extremality of $v_{-}$and $u_{+}$, we have that $y_{0} \in C_{0}^{1}(\bar{\Omega})$ is nodal.

In the next section, we will use this result in the framework of semilinear equations (i.e., $p=2$ ) to produce one more nontrivial smooth solution.

\section{Semilinear Problem}

In this section, we deal with the case $p=2$ (semilinear equation). So, the problem under consideration is the following:

$$
\left\{\begin{array}{l}
-\Delta u(z)=\lambda|u(z)|^{q-2} u(z)+f(z, u(z)) \quad \text { in } \Omega \\
\left.u\right|_{\partial \Omega}=0,1<q<2, \lambda>0
\end{array}\right.
$$

In what follows $\left\{\lambda_{k}\right\}_{k \geqslant 1}$ are the distinct eigenvalues of $-\Delta^{D}$. The hypotheses on $f(z, \zeta)$ are the following: 
$\underline{H(f)_{4}} \quad f: \Omega \times \mathrm{R} \longrightarrow \mathrm{R}$ is a function, such that:

(i) for all $\zeta \in \mathbf{R}$, the function $z \longmapsto f(z, \zeta)$ is measurable;

(ii) for almost all $z \in \Omega$, the function $\zeta \longmapsto f(z, \zeta)$ is $C^{1}, f(z, 0)=0$;

(iii) there exist $a \in L^{\infty}(\Omega)_{+}, c>0$ and $2<r<2^{*}$, such that

$$
\left|f_{\zeta}^{\prime}(z, \zeta)\right| \leqslant a(z)+c|\zeta|^{r-2} \quad \text { for almost all } z \in \Omega \text { and all } \zeta \in \mathrm{R}
$$

(iv) there exist integer $m \geqslant 2$ and functions $\eta_{1}, \eta_{2} \in L^{\infty}(\Omega)_{+}$, such that $\eta_{1} \neq \lambda_{m}, \eta_{2} \neq \lambda_{m+1}$,

$$
\lambda_{m} \leqslant \eta_{1}(z) \leqslant \eta_{2}(z) \leqslant \lambda_{m+1} \quad \text { for almost all } z \in \Omega
$$

and

$$
\eta_{1} \leqslant \liminf _{|\zeta| \rightarrow+\infty} \frac{f(z, \zeta)}{\zeta} \leqslant \limsup _{|\zeta| \rightarrow+\infty} \frac{f(z, \zeta)}{\zeta} \leqslant \eta_{2}(z)
$$

uniformly for almost all $z \in \Omega$;

(v) there exists $\vartheta \in L^{\infty}(\Omega)_{+}$, such that $\varrho \neq \lambda_{1}$,

$$
\vartheta(z) \leqslant \lambda_{1} \quad \text { for almost all } z \in \Omega
$$

and

$$
\limsup _{\zeta \rightarrow 0} \frac{2 F(z, \zeta)}{\zeta^{2}} \leqslant \vartheta(z)
$$

uniformly for almost all $z \in \Omega$ and

$$
f(z, \zeta) \zeta \geqslant 0 \quad \text { for almost all } z \in \Omega \text {, all } \zeta \in \mathbf{R}
$$

(vi) there exist $\mu, r>0$ and $\hat{c}_{0}>0$, such that $q \leqslant \mu<2<r$ and

$$
\mu F(z, \zeta)-f(z, \zeta) \zeta \geqslant-\hat{c}_{0}|\zeta|^{r} \quad \text { for almost all } z \in \Omega \text {, all } \zeta \in \mathrm{R} .
$$

EXAMPLE 5.1. The following function $f(\zeta)$ satisfies hypotheses $H(f)_{4}$ (again for the sake of simplicity we drop the $z$-dependence):

$$
f(\zeta)= \begin{cases}\eta|\zeta|^{\tau-2} \zeta-\zeta+|\zeta|^{q-2} \zeta & \text { if }|\zeta| \leqslant 1, \\ \eta \zeta & \text { if }|\zeta|>1,\end{cases}
$$

with $\eta \in\left(\lambda_{m}, \lambda_{m+1}\right), m \geqslant 1,1<\tau<2<q$.

In what follows by $E\left(\lambda_{k}\right)$ we denote the eigenspace corresponding to the eigenvalues $\lambda_{k}>0$. 
Theorem 5.2. If hypotheses $H(f)_{4}$ hold, $\lambda^{*}$ is as postulated in Proposition 3.7 and $\lambda \in\left(0, \lambda^{*}\right)$, then problem (5.1) has at least six nontrivial smooth solutions

$$
\begin{gathered}
u_{0}, \hat{u} \in \operatorname{int} C_{+}, \quad v_{0}, \hat{v} \in-\operatorname{int} C_{+}, \quad y_{0}, w_{0} \in C_{0}^{1}(\bar{\Omega}) \text { nodal with } \\
u_{0}-y_{0}, \hat{u}-y_{0} \in \operatorname{int} C_{+}, \quad y_{0}-v_{0}, y_{0}-\hat{v} \in-\operatorname{int} C_{+} .
\end{gathered}
$$

Proof. From Theorem 4.3, we already have five nontrivial smooth solutions

$$
u_{0}, \hat{u} \in \operatorname{int} C_{+}, \quad v_{0}, \hat{v} \in-\operatorname{int} C_{+} \quad \text { and } \quad y_{0} \in C_{0}^{1}(\bar{\Omega}) \text { nodal. }
$$

Also, by virtue of hypotheses $H(f)_{4}$ (ii) and (iii), we can find $c_{7}>0$, such that for almost all $z \in \Omega$,

the function $[-\beta, \beta] \ni \zeta \longmapsto f(z, \zeta)+c_{7} \zeta$ is nondecreasing,

with $\beta=\max \left\{\left\|v_{-}\right\|_{\infty},\left\|u_{+}\right\|_{\infty}\right\}$, where $v_{-}$and $u_{+}$are the extremal solutions postulated in Proposition 3.12. Then, recalling that $y_{0} \in\left[v_{-}, u_{+}\right]$, for almost all $z \in \Omega$, we have

$$
\begin{aligned}
-\Delta y_{0}(z)+c_{7} y_{0}(z) & =f\left(z, y_{0}(z)\right)+c_{7} y_{0}(z) \\
& \leqslant f\left(z, u_{+}(z)\right)+c_{7} u_{+}(z) \\
& =-\Delta u_{+}(z)+c_{7} u_{+}(z),
\end{aligned}
$$

so

$$
\Delta\left(u_{+}-y_{0}\right)(z) \leqslant c_{7}\left(u_{+}-y_{0}\right)(z) \quad \text { for almost all } z \in \Omega .
$$

Invoking the strong maximum principle of Vázquez [26], we obtain

$$
u_{+}-y_{0} \in \operatorname{int} C_{+} .
$$

In a similar fashion, we show that

$$
y_{0}-v_{-} \in \operatorname{int} C_{+} .
$$

Therefore, due to the extremality of $u_{+}$and $v_{-}$, we have

$$
u_{0}-y_{0}, \hat{u}-y_{0} \in \operatorname{int} C_{+}, \quad y_{0}-v_{0}, y_{0}-\hat{v} \in-\operatorname{int} C_{+} .
$$

Then from Liu-Wu [20], we know that

$$
C_{k}\left(\left.\hat{\varphi}^{\lambda}\right|_{C_{0}^{1}(\bar{\Omega})}, y_{0}\right)=C_{k}\left(\hat{\varphi}^{\lambda}, y_{0}\right) \quad \forall k \geqslant 0
$$


and

$$
C_{k}\left(\left.\varphi^{\lambda}\right|_{C_{0}^{1}(\bar{\Omega})}, y_{0}\right)=C_{k}\left(\varphi^{\lambda}, y_{0}\right) \quad \forall k \geqslant 0 .
$$

From (5.2) and (5.3), we see that $u_{0} \in \operatorname{int}_{C_{0}^{1}(\bar{\Omega})}\left[v_{-}, u_{+}\right]$. Also note that

$$
\left.\hat{\varphi}_{\lambda}\right|_{\left[v_{-}, u_{+}\right]}=\left.\varphi^{\lambda}\right|_{\left[v_{-}, u_{+}\right]} \text {. }
$$

So from the definition of critical groups, we have

$$
C_{k}\left(\left.\hat{\varphi}^{\lambda}\right|_{C_{0}^{1}(\bar{\Omega})}, y_{0}\right)=C_{k}\left(\left.\varphi^{\lambda}\right|_{C_{0}^{1}(\bar{\Omega})}, y_{0}\right) \quad \forall k \geqslant 0,
$$

so, from (5.4) and (5.5), we have

$$
C_{k}\left(\hat{\varphi}^{\lambda}, y_{0}\right)=C_{k}\left(\varphi^{\lambda}, y_{0}\right) \quad \forall k \geqslant 0 .
$$

We have $\hat{\varphi}^{\lambda} \in C^{2-0}\left(H_{0}^{1}(\Omega)\right)$. Suppose that the spectrum of $\left(\hat{\varphi}^{\lambda}\right)^{\prime \prime}\left(y_{0}\right)$ is in $[0,+\infty)$. Then

$$
\|\nabla u\|_{2}^{2} \geqslant \int_{\Omega} m u^{2} d z \quad \forall u \in H_{0}^{1}(\Omega),
$$

where $m(\cdot)=f_{\zeta}^{\prime}\left(\cdot, y_{0}(\cdot)\right) \in L^{\infty}(\Omega)$. If $u \in \operatorname{ker}\left(\hat{\varphi}^{\lambda}\right)^{\prime \prime}\left(y_{0}\right)$ and $m^{+}=0$, then $u=0$. If $u \in \operatorname{ker}\left(\hat{\varphi}^{\lambda}\right)^{\prime \prime}\left(y_{0}\right)$ and $m^{+} \neq 0$, then principal eigenvalue of the weighted problem

$$
\left\{\begin{array}{l}
-\Delta u(z)=\hat{\lambda} m u \quad \text { in } \Omega \\
\left.u\right|_{\partial \Omega}=0
\end{array}\right.
$$

is given by

$$
\hat{\lambda}_{1}(m)=\inf \left\{\|\nabla u\|_{2}^{2}: \int_{\Omega} m u^{2} d z=1, u \in H_{0}^{1}(\Omega)\right\},
$$

so $\hat{\lambda}_{1}(m) \geqslant 1$ and it is simple, so

$$
\operatorname{dim} \operatorname{ker}\left(\hat{\varphi}^{\lambda}\right)^{\prime \prime}\left(y_{0}\right) \leqslant 1 .
$$

Hence, from Theorem 2.7 of Li-Li-Liu [18], we have

$$
C_{k}\left(\hat{\varphi}^{\lambda}, y_{0}\right)=\delta_{k, 1} \mathrm{Z} \quad \forall k \geqslant 0,
$$

so, using (5.6), we have

$$
C_{k}\left(\varphi^{\lambda}, y_{0}\right)=\delta_{k, 1} \mathrm{Z} \quad \forall k \geqslant 0 .
$$


Similarly, $\left\{u_{0}, v_{0}\right\}$ are critical points of mountain pass type for the functional $\varphi^{\lambda}$ and by virtue of hypotheses $H(f)_{4}, \varphi^{\lambda} \in C^{2}\left(H_{0}^{1}(\Omega) \backslash\{0\}\right)$. Hence

$$
C_{k}\left(\varphi^{\lambda}, u_{0}\right)=C_{k}\left(\varphi^{\lambda}, v_{0}\right)=\delta_{k, 1} z \quad \forall k \geqslant 0 .
$$

Moreover, $\hat{u}, \hat{v}$ are local minimizers of $\varphi^{\lambda}$ and so

$$
C_{k}\left(\varphi^{\lambda}, \hat{u}\right)=C_{k}\left(\varphi^{\lambda}, \hat{v}\right)=\delta_{k, 0} \mathrm{Z} \quad \forall k \geqslant 0 .
$$

From Proposition 4.2, we have

$$
C_{k}\left(\varphi^{\lambda}, 0\right)=0 \quad \forall k \geqslant 0 .
$$

Finally we need to compute the critical groups of $\varphi^{\lambda}$ at infinity. To this end, we consider the homotopy

$$
h_{\lambda}(t, u)=(1-t) \varphi^{\lambda}(u)+t \psi(u) \quad \forall(t, u) \in[0,1] \times H_{0}^{1}(\Omega),
$$

where $\psi: H_{0}^{1}(\Omega) \longrightarrow \mathrm{R}$ is the $C^{2}$-functional, defined by

$$
\psi(u)=\frac{1}{2}\|\nabla u\|_{2}^{2}-\frac{1}{2} \int_{\Omega} g_{0} u^{2} d z,
$$

with $g_{0} \in L^{\infty}(\Omega)_{+}, \eta_{1} \leqslant g_{0} \leqslant \eta_{2}$. Suppose that we can find two sequences $\left\{t_{n}\right\}_{n \geqslant 1} \subseteq[0,1]$ and $\left\{u_{n}\right\}_{n \geqslant 1} \subseteq H_{0}^{1}(\Omega)$, such that

$$
t_{n} \longrightarrow t \quad \text { in }[0,1], \quad\left\|u_{n}\right\| \longrightarrow+\infty
$$

and

$$
\left(h_{\lambda}\right)_{u}^{\prime}\left(t_{n}, u_{n}\right) \longrightarrow 0
$$

So,

$$
\begin{aligned}
\mid\left\langle A\left(u_{n}\right), h\right\rangle-\left(1-t_{n}\right) & \left(\lambda \int_{\Omega}\left|u_{n}\right|^{q-2} u_{n} h d z-\int_{\Omega} f\left(z, u_{n}\right) h d z\right) \\
& -t_{n} \int_{\Omega} g_{0} u_{n} h d z \mid \leqslant \varepsilon_{n}\|h\| \quad \forall h \in H_{0}^{1}(\Omega),
\end{aligned}
$$

with $\varepsilon_{n} \searrow 0$. Let

$$
y_{n}=\frac{u_{n}}{\left\|u_{n}\right\|} \quad \forall n \geqslant 1 .
$$

Then $\left\|y_{n}\right\|=1$ for all $n \geqslant 1$ and so we may assume that

$$
\begin{array}{ll}
y_{n} \longrightarrow y & \text { weakly in } H_{0}^{1}(\Omega), \\
y_{n} \longrightarrow y & \text { in } L^{2}(\Omega) .
\end{array}
$$


From (5.13), we have

$$
\begin{array}{r}
\mid\left\langle A\left(y_{n}\right), h\right\rangle-\left(1-t_{n}\right)\left(\lambda \int_{\Omega}\left|u_{n}\right|^{q-2} y_{n} h d z-\int_{\Omega} \frac{f\left(z, u_{n}\right)}{\left\|u_{n}\right\|} h d z\right) \\
-t_{n} \int_{\Omega} g_{0} y_{n} h d z \mid \leqslant \frac{\varepsilon_{n}}{\left\|u_{n}\right\|}\|h\| \quad \forall h \in H_{0}^{1}(\Omega) .
\end{array}
$$

Note that

$$
\left|u_{n}(z)\right| \longrightarrow+\infty \quad \text { for almost all } z \in\{y \neq 0\}
$$

Hence, since $q \in(1,2)$, we have

$$
\int_{\Omega}\left|u_{n}\right|^{q-2} y_{n} h d z \longrightarrow 0 .
$$

Also, we have

$$
\frac{f\left(\cdot, u_{n}(\cdot)\right)}{\left\|u_{n}\right\|} \longrightarrow g y \quad \text { weakly in } L^{2}(\Omega),
$$

where $g \in L^{2}(\Omega)_{+}, \eta_{1} \leqslant g \leqslant \eta_{2}$ (see Motreanu-Motreanu-Papageorgiou [22]). So, if in (5.14) we pass to the limit as $n \rightarrow+\infty$, then

$$
\langle A(y), h\rangle=\int_{\Omega} g_{t} y h d z \quad \forall h \in H_{0}^{1}(\Omega),
$$

where $g_{t}=(1-t) g+t g_{0}$, so

$$
A(y)=g_{t} y
$$

and thus

$$
\left\{\begin{array}{l}
-\Delta y(z)=g_{t}(z) y(z) \quad \text { in } \Omega \\
\left.y\right|_{\partial \Omega}=0
\end{array}\right.
$$

Note that $\eta_{1} \leqslant g_{t} \leqslant \eta_{2}$ and by virtue of the unique continuation principle and hypothesis $H(f)_{4}$ (iv), we have

$$
\hat{\lambda}_{m}\left(g_{t}\right)<\hat{\lambda}_{m}\left(\lambda_{m}\right)=1 \quad \text { and } \quad \hat{\lambda}_{m+1}\left(g_{t}\right)>\hat{\lambda}_{m+1}\left(\lambda_{m+1}\right)=1 .
$$

Then from (5.15) and (5.16), we infer that $y=0$.

Next on (5.14) we choose $h=y_{n}-y \in H_{0}^{1}(\Omega)$ and pass to the limit as $n \rightarrow+\infty$. We obtain

$$
\lim _{n \rightarrow+\infty}\left\langle A\left(y_{n}\right), y_{n}-y\right\rangle=0,
$$


so, from Proposition 2.4, we have

$$
y_{n} \longrightarrow y \quad \text { in } H_{0}^{1}(\Omega)
$$

and so $\|y\|=1$, a contradiction.

Therefore, there are no sequences $\left\{t_{n}\right\}_{n} \geqslant 1 \subseteq[0,1]$ and $\left\{u_{n}\right\}_{n} \geqslant 1 \subseteq H_{0}^{1}(\Omega)$ for which (5.12) is true. So, we can apply Lemma 2.4 of Perera-Schechtera [25] and have

$$
C_{k}\left(\varphi^{\lambda}, \infty\right)=C_{k}(\psi, \infty) \quad \forall k \geqslant 0 .
$$

Since $\eta_{1} \leqslant g_{0} \leqslant \eta_{2}, u=0$ is the only critical point of $\psi$ and so

$$
C_{k}(\psi, \infty)=C_{k}(\psi, 0) \quad \forall k \geqslant 0 .
$$

But $u=0$ is a nondegenerate critical point of $\psi$ with Morse index

$$
d_{m}=\operatorname{dim} \bigoplus_{k=1}^{m} E\left(\lambda_{k}\right)
$$

(see e.g., Gasiński-Papageorgiou [9, p. 718]). Therefore

$$
C_{k}(\psi, 0)=\delta_{k, d_{m}} Z \quad \forall k \geqslant 0 .
$$

Combining (5.17), (5.18) and (5.19), we infer that

$$
C_{k}\left(\varphi^{\lambda}, \infty\right)=\delta_{k, d_{m}} \mathrm{z} \quad \forall k \geqslant 0 .
$$

Suppose that $\left\{0, u_{0}, \hat{u}, v_{0}, \hat{v}, y_{0}\right\}$ are the only critical points of $\varphi^{\lambda}$. Otherwise, we already have a sixth nontrivial smooth (by regularity theory) solution of (5.1). Then, from (5.7), (5.8), (5.9), (5.10), (5.20) and the Morse relation (2.2), we have

$$
2+3 t=t^{d_{m}}+(1+t) Q(t)
$$

a contradiction, since $d_{m} \geqslant 2$.

This means that $\varphi^{\lambda}$ has one more critical point $w_{0} \notin\left\{0, u_{0}, \hat{u}, v_{0}, \hat{v}, y_{0}\right\}$, which is the sixth nontrivial solution of (5.1) and standard regularity theory implies $w_{0} \in C_{0}^{1}(\bar{\Omega})$

\section{The Critical Case $\lambda=\lambda^{*}$}

In this section we examine what happens in the critical case $\lambda=\lambda^{*}$. In the next theorem we show that, if $\lambda=\lambda^{*}$, then we can still guarantee two nontrivial solutions of constant sign, one positive and the other negative. 
THEOREM 6.1.

(a) If hypotheses $H(f)_{2}$ hold and $\lambda=\lambda_{+}^{*}$, then problem (1.1) has at least one positive smooth solution $u_{0} \in \operatorname{int} C_{+}$.

(b) If hypotheses $H(f)_{2}$ hold and $\lambda=\lambda_{-}^{*}$, then problem (1.1) has at least one negative smooth solution $v_{0} \in-\operatorname{int} C_{+}$. smallskip

(c) If hypotheses $H(f)_{2}$ hold and $\lambda=\lambda^{*}=\min \left\{\lambda_{+}^{*}, \lambda_{-}^{*}\right\}$, then problem (1.1) has at least two nontrivial constant sign smooth solutions $u_{0} \in$ int $C_{+}, v_{0} \in-\operatorname{int} C_{+}$.

Proof. (a) Let $\lambda_{n}<\lambda_{+}^{*}$ for all $n \geqslant 1$ and suppose that

$$
\lambda_{n} \longrightarrow \lambda_{+}^{*} \text {. }
$$

From Proposition 3.7(a), we know that problem (1.1), with $\lambda=\lambda_{n}$, has a solution $u_{0}^{n} \in \operatorname{int} C_{+}$for $n \geqslant 1$. Then

$$
A\left(u_{0}^{n}\right)=\lambda_{n}\left(u_{0}^{n}\right)^{q-1}+N\left(u_{0}^{n}\right) \quad \forall n \geqslant 1 .
$$

Suppose that $\left\|u_{0}^{n}\right\| \longrightarrow+\infty$ as $n \rightarrow+\infty$. We set

$$
y_{n}=\frac{u_{0}^{n}}{\left\|u_{0}^{n}\right\|} \quad \forall n \geqslant 1 .
$$

Since $\left\|y_{n}\right\|=1$ for all $n \geqslant 1$, passing to a subsequence if necessary, we may assume that

$$
\begin{array}{ll}
y_{n} \longrightarrow y & \text { weakly in } W_{0}^{1, p}(\Omega), \\
y_{n} \longrightarrow y & \text { in } L^{p}(\Omega) .
\end{array}
$$

From (6.1), we have

$$
A\left(y_{n}\right)=\lambda_{n} \frac{1}{\left\|u_{n}\right\|^{p-q}} y_{n}^{q-1}+\frac{N\left(u_{0}^{n}\right)}{\left\|u_{n}\right\|^{p-1}} \quad \forall n \geqslant 1 .
$$

Acting on (6.4) with $y_{n}-y \in W_{0}^{1, p}(\Omega)$ and then passing to the limit as $n \rightarrow+\infty$, we obtain

$$
\lim _{n \rightarrow+\infty}\left\langle A\left(y_{n}\right), y_{n}-y\right\rangle=0
$$

(recall that $q<p$ ) and using Proposition 2.4, we have

$$
y_{n} \longrightarrow y \quad \text { in } W_{0}^{1, p}(\Omega),
$$


hence $\|y\|=1$. We know that

$$
\frac{N\left(u_{0}^{n}\right)}{\left\|u_{0}^{n}\right\|^{p-1}} \longrightarrow g y^{p-1} \quad \text { weakly in } W_{0}^{1, p}(\Omega),
$$

where $g \in L^{\infty}(\Omega)_{+}, \eta_{1} \leqslant g \leqslant \eta_{2}$ (see the proof of Proposition 3.3 and Perera [24]). So, if in (6.4) we pass to the limit as $n \rightarrow+\infty$ and we use (6.5), (6.6) and the fact that $q<p$, we obtain

$$
A(y)=g y^{p-1},
$$

so

$$
\left\{\begin{array}{l}
-\Delta_{p} y(z)=g(z) y(z) \quad \text { in } \Omega, \\
\left.y\right|_{\partial \Omega}=0, y \geqslant 0, y \neq 0 .
\end{array}\right.
$$

But by virtue of hypothesis $H(f)_{2}$ (iv), $\hat{\lambda}_{1}(g)<\hat{\lambda}_{1}\left(\lambda_{1}\right)=1$ and so $y$ must change sign, a contradiction to (6.7). This proves that the sequence $\left\{u_{0}^{n}\right\}_{n \geqslant 1} \subseteq$ $H_{0}^{1}(\Omega)$ is bounded and so we may assume that

$$
\begin{array}{ll}
u_{0}^{n} \longrightarrow u_{0} & \text { weakly in } W_{0}^{1, p}(\Omega), \\
u_{0}^{n} \longrightarrow u_{0} & \text { in } L^{p}(\Omega) .
\end{array}
$$

Acting on (6.1) with $u_{0}^{n}-u_{0} \in W_{0}^{1, p}(\Omega)$, passing to the limit as $n \rightarrow+\infty$ and using Proposition 2.4, we obtain

$$
u_{0}^{n} \longrightarrow u_{0} \quad \text { in } W_{0}^{1, p}(\Omega),
$$

hence $u_{0} \geqslant 0$. We may assume that $0<\beta \leqslant \lambda_{n}$ for all $n \geqslant 1$. Choose $\varepsilon>0$ small enough, such that

$$
\lambda_{1} \varepsilon^{p-1} \hat{u}_{1}(z)^{p-1}<\beta \varepsilon^{q-1} \hat{u}_{1}(z)^{q-1} \quad \forall z \in \Omega .
$$

If we set $\underline{u}=\varepsilon \hat{u}_{1} \in \operatorname{int} C_{+}$. Then from the proof of Proposition 3.12, we know that we can assume that $\underline{u} \leqslant u_{0}^{n}$ for all $n \geqslant 1$. So, $\underline{u} \leqslant u_{0}$, hence $u_{0} \neq 0$.

Passing to the limit as $n \rightarrow+\infty$ in (6.1) and using (6.8), we obtain

$$
A\left(u_{n}\right)=\lambda_{+}^{*} u_{0}^{q-1}+N\left(u_{0}\right),
$$

so

$$
\left\{\begin{array}{l}
-\Delta_{p} u_{0}(z)=\lambda_{+}^{*} u_{0}(z)^{q-1}+f\left(z, u_{0}(z)\right) \quad \text { in } \Omega, \\
\left.u_{0}\right|_{\partial \Omega}=0, u_{0} \neq 0,
\end{array}\right.
$$

so $u_{0} \in \operatorname{int} C_{+}$(nonlinear regularity theory). 
(b) The proof of this part is similar to that of (b) and so it is omitted.

(c) Combining (a) and (b), we have (c).

Again in the semilinear case $(p=2)$ and using hypotheses $H(f)_{4}$, we can have more solutions.

THeOREM 6.2. If hypotheses $H(f)_{4}$ hold and $\lambda=\lambda^{*}=\min \left\{\lambda_{+}^{*}, \lambda_{-}^{*}\right\}$, then problem (1.1) has at least three nontrivial smooth solutions

$$
u_{0} \in \operatorname{int} C_{+}, \quad v_{0} \in-\operatorname{int} C_{+}, \quad \text { and } \quad y_{0} \in C_{0}^{1}(\bar{\Omega}) .
$$

Proof. From Theorem 6.1, we already have two nontrivial constant sign solutions

$$
u_{0} \in \operatorname{int} C_{+} \quad \text { and } \quad v_{0} \in-\operatorname{int} C_{+} .
$$

From the proof of Theorem 6.1, we know that

$$
u_{0}^{n} \longrightarrow u_{0} \quad \text { and } \quad v_{0}^{n} \longrightarrow v_{0} \quad \text { in } H_{0}^{1}(\Omega),
$$

where $u_{0}^{n}$ and $v_{0}^{n}$ are critical points of $\varphi^{\lambda_{n}}$ of mountain pass type (here $\lambda_{n}<\lambda^{*}$ for all $n \geqslant 1$ and $\lambda_{n} \longrightarrow \lambda^{*}$ ). So, we have

$$
C_{k}\left(\varphi^{\lambda_{n}}, u_{0}^{n}\right)=C_{k}\left(\varphi^{\lambda_{n}}, v_{0}^{n}\right)=\delta_{k, 1} z \quad \forall k \geqslant 0, n \geqslant 1 .
$$

Since $\varphi^{\lambda_{n}} \longrightarrow \varphi^{\lambda^{*}}$ in $C^{1}\left(H_{0}^{1}(\Omega)\right)$ as $n \rightarrow+\infty$, exploiting the continuity of the critical groups on the $C^{1}$-norm (see Chang [2, p. 336]), we have

$$
C_{k}\left(\varphi^{\lambda^{*}}, u_{0}\right)=C_{k}\left(\varphi^{\lambda^{*}}, v_{0}\right)=\delta_{k, 1} \mathrm{Z} \quad \forall k \geqslant 0 .
$$

From Proposition 4.2, we know that

$$
C_{k}\left(\varphi^{\lambda^{*}}, 0\right)=0 \quad \forall k \geqslant 0 .
$$

Finally, as in the proof of Theorem 5.2, using Lemma 2.4 of Perera-Schechtera [25], we obtain

$$
C_{k}\left(\varphi^{\lambda^{*}}, \infty\right)=\delta_{k, d_{m}} \mathrm{z} \quad \forall k \geqslant 0 .
$$

Suppose that $\left\{0, u_{0}, v_{0}\right\}$ are the only critical points of $\varphi^{\lambda^{*}}$ (otherwise we are done). Then, from (6.9), (6.10), (6.11) and the Morse relation (2.2) with $t=$ -1 , we have

$$
2(-1)^{1}=(-1)^{d_{m}}
$$

a contradiction.

This proves that $\varphi^{\lambda^{*}}$ has one more critical point $y_{0} \notin\left\{0, u_{0}, v_{0}\right\}$. This solves problem (5.1) and regularity theory implies that $y_{0} \in C_{0}^{1}(\bar{\Omega})$. 
REMARK 6.3. It is an interesting open question whether $y_{0} \in C_{0}^{1}(\bar{\Omega})$ can be shown to be a nodal solution.

ACKNOWLEDGement. The authors wish to thank a knowledgeable referee for their corrections and remarks that improved the paper considerably.

\section{REFERENCES}

1. Ambrosetti, A., Brézis, H., and Cerami, G., Combined effects of concave and convex nonlinearities in some elliptic problems, J. Funct. Anal. 122 (1994), 519-543.

2. Chang, K.-C., Methods in nonlinear analysis, Springer, Berlin 2005.

3. Clarke, F. H., Optimization and nonsmooth analysis, Wiley, New York 1983.

4. de Paiva, F. O., and Massa, E., Multiple solutions for some elliptic equations with a nonlinearity concave at the origin, Nonlinear Anal. 66 (2007), 2940-2946.

5. Dunford, H., and Schwartz, J.T., Linear Operators. I. General Theory, Pure and Appl. Math. 7, Interscience, London 1958.

6. Filippakis, M., Gasiński, L., and Papageorgiou, N.S., On the existence of positive solutions for hemivariational inequalities driven by the p-Laplacian, J. Global Optim. 31 (2005), 173-189.

7. Filippakis, M., Kristaly, A., and Papageorgiou, N. S., Existence of five nontrivial solutions with precise sign data for a p-Laplacian equation, Discrete Contin. Dyn. Syst. 24 (2009), 405-440.

8. García Azorero, J., Manfredi, J., and Peral Alonso, I., Sobolev versus Hölder local minimizers and global multiplicity for some quasilinear elliptic equations, Commun. Contemp. Math. 2 (2000), 385-404.

9. Gasiński, L., and Papageorgiou, N. S., Nonlinear Analysis, Chapman and Hall, Boca Raton 2006.

10. Gasiński, L., and Papageorgiou, N. S., Nodal and multiple constant sign solutions for resonant p-laplacian equations with a nonsmooth potential, Nonlinear Anal. 71 (2009), 5747-5772.

11. Gasiński, L., and Papageorgiou, N. S., Multiple solutions for nonlinear Neumann problems with asymmetric reaction, via Morse theory, Adv. Nonlinear Stud. 11 (2011), 781-808.

12. Gasiński, L., and Papageorgiou, N. S., Multiple solutions for asymptotically ( $p-1)$-homogeneous p-Laplacian equations, J. Funct. Anal. 262 (2012), 2403-2435.

13. Gasiński, L., and Papageorgiou, N. S. Dirichlet problems with double resonance and an indefinite potential, Nonlinear Anal. 75 (2012), 4560-4595.

14. Granas, A., and Dugundji, J., Fixed Point Theory, Springer, Berlin 2003.

15. Guedda, M., and Véron, L., Quasilinear elliptic equations involving critical Sobolev exponents, Nonlinear Anal. 13 (1989), 879-902.

16. Guo, Z., and Zhang, Z., $W^{1, p}$ versus $C^{1}$ Local minimizers and multiplicity results for quasilinear elliptic equations, J. Math. Anal. Appl. 286 (2003), 32-50.

17. Kyritsi, S. T., and Papageorgiou, N. S., Pairs of positive solutions for p-Laplacian equations with combined nonlinearities, Comm. Pure Appl. Anal. 8 (2009), 1031-1051.

18. Li, C., Li, S., and Liu, J., Splitting theorem, Poincaré-Hopf theorem and jumping nonlinear problems, J. Funct. Anal. 221 (2005), 439-455.

19. Li, S.-J., Wu, S., and Zhou, H.S., Solutions to semilinear elliptic problems with combined nonlinearities, J. Differential Equations 185 (2002), 200-224.

20. Liu, J.-Q., and Wu, S., Calculating critical groups of solutions for elliptic problems with jumping nonlinearity, Nonlinear Anal. 49 (2002), 779-797.

21. Moroz, V., Solutions of superlinear at zero elliptic equations via Morse theory, Topol. Methods Nonlinear Anal. 10 (1997), 387-397. 
22. Motreanu, D., Motreanu, V. V., and Papageorgiou, N. S., A degree theoretic approach for multiple solutions of constant sign for nonlinear elliptic equations, Manuscripta Math. 124 (2007), 507-531.

23. Papageorgiou, N. S., and Rocha, E. M., Existence of three nontrivial solutions for asymptotically p-linear noncoercive p-Laplacian equations, Nonlinear Anal. 74 (2011), 5314-5326.

24. Perera, K., Multiplicity result for some elliptic problems with concave nonlinearity, J. Differential Equations 140 (1997), 133-141.

25. Perera, K., and Schechter, M., Solutions of nonlinear equations having asymptotic limits at zero and infinity, Calc. Var. Partial Differential Equations 12 (2001), 359-369.

26. Vázquez, J. L., A strong maximum principle for some quasilinear elliptic equations, Appl. Math. Optim. 12 (1984), 191-202.

27. Wang, Z.-Q., Nonlinear boundary value problems with concave nonlinearities near the origin, NoDEA Nonlinear Differential Equations Appl. 8 (2001), 15-33.

28. Wu, S. P., and Yang, H., A class of resonant elliptic problems with sublinear nonlinearity at origin and at infinity, Nonlinear Anal. 45 (2001), 925-935.

JAGIELLONIAN UNIVERSITY

FACULTY OF MATHEMATICS AND COMPUTER SCIENCE

ul. ŁOJASIEWICZA 6

30-348 KRAKÓW

POLAND

E-mail: Leszek.Gasinski@ii.uj.edu.pl
NATIONAL TECHNICAL UNIVERSITY DEPARTMENT OF MATHEMATICS ZOGRAFOU CAMPUS ATHENS 15780

GREECE

E-mail: npapg@math.ntua.gr 